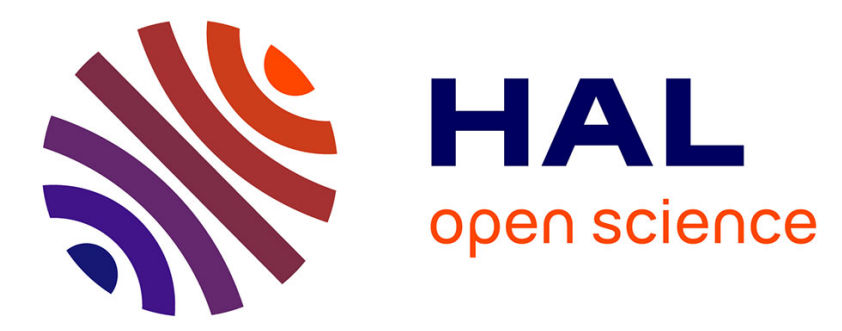

\title{
The Strategic Sincerity of Approval Voting
}

Matias Nunez

\section{To cite this version:}

Matias Nunez. The Strategic Sincerity of Approval Voting. Economic Theory, 2013, pp.0938-2259. 10.1007/s00199-013-0775-x . hal-00917101

\section{HAL Id: hal-00917101 \\ https://hal.science/hal-00917101}

Submitted on 11 Dec 2013

HAL is a multi-disciplinary open access archive for the deposit and dissemination of scientific research documents, whether they are published or not. The documents may come from teaching and research institutions in France or abroad, or from public or private research centers.
L'archive ouverte pluridisciplinaire HAL, est destinée au dépôt et à la diffusion de documents scientifiques de niveau recherche, publiés ou non, émanant des établissements d'enseignement et de recherche français ou étrangers, des laboratoires publics ou privés. 


\title{
The Strategic Sincerity of Approval Voting *
}

\author{
Matías Núñez ${ }^{\dagger}$
}

JUNE 2013

\begin{abstract}
We show that Approval Voting need not trigger sincere behavior in equilibrium of Poisson voting games and hence might lead a strategic voter to skip a candidate preferred to her worst preferred approved candidate. We identify two main rationales for these violations of sincerity. First, if a candidate has no votes, a voter might skip him. Notwithstanding, we provide sufficient conditions on the voters' preference intensities to remove this sort of insincerity. On the contrary, if the candidate gets a positive share of the votes, a voter might skip him solely on the basis of her ordinal preferences. This second type of insincerity is a consequence of the correlation of the candidates' scores. The incentives for sincerity of rank scoring rules are also discussed.
\end{abstract}

KEYWORDS: Sincerity, Approval Voting, Poisson Games.

JEL Classification Numbers: D72.

\section{Introduction}

Among the different properties of Plurality Voting $(P V)$, the wasted-vote effect is among the most undesirable ones. For instance, if a rational voter believes that a

\footnotetext{
${ }^{*}$ I wish to thank Steve Brams, Sébastien Courtin, Arnaud Dellis, Francesco De Sinopoli, Fabian Gouret, Laurence Jacquet, Jean-François Laslier, François Maniquet, Mathieu Martin and Klaus Nehring for their valuable comments. I would also like to thank the editor, Nicholas Yannelis, and two referees for their excellent suggestions and comments that substantially upgraded the quality of this work. This article was previously circulated under the title "Sincere Scoring Rules" [34]. This research has been developed within the center of excellence MME-DII (ANR-11-LBX-0023-01).

${ }^{+}$Université de Cergy-Pontoise, CNRS, THEMA, F-95000 Cergy-Pontoise, France. E-mail address: matias.nunez@u-cergy.fr
} 
candidate gets no vote, then she does not vote for him as it is dominant to vote for one of the likely-winners of the election. The main problem is that if this candidate is the voter's preferred one, these beliefs lead to a rational violation of sincerity. In order to overcome such an effect, one may focus on the Approval Voting method $(A V)$. As argued by Merril and Nagel [24], "much of the case for Approval [Voting] hinges on its encouragement of sincere voting". Indeed, under $A V$, a voter is allowed to approve of as many candidates as she wants, the winner(s) being the candidate(s) with the most votes ${ }^{1}$. This method is hence immune to the previous effect with just three candidates. Indeed, as the voter can approve of as many candidates as she wants, "dominance implies that everyone gives an approval point to his favorite candidate, and no one gives an approval point to his worst candidate"(Myerson [28]). Hence, with just three candidates, the voter will either vote for her preferred candidate or for her two preferred-ones. In other words, the voter does not skip a candidate: she votes for all the candidates ranked above some candidate. This nice feature is often referred to as the no-skipping sincerity of $A V$ and it is the most used notion among the different sincerity definitions with this rule. Indeed, there are different notions of sincerity with this method (discussed in Section 4) as it breaks the one-to-one correspondence between the sets of ordinal preference orderings and the set of ballots inherent to the Arrovian tradition (and hence present in rules as $P V$ or the Borda rule). We focus on the sincerity under $A V$ with more than three candidates in a strategic framework in which the voters' beliefs are endogenous since they depend on the voter's behavior.

We prove that, in general, $A V$ need not trigger (any kind of) sincere behavior in equilibrium in Poisson voting games. In these games there is a random number of voters and were proposed by Myerson $[27,28]^{2}$ to study strategic voting. We discuss two examples (Examples 1 and 2) that prove that it might be a strict best response to violate the different notions of sincerity in these games. These examples are counterintuitive specially when compared to the several statements in the literature that suggest that the voters always have a sincere best response under $A V$ in any pure strategy equilibrium. Among them, Niemi [31], states that "under $A V$, voters are never urged to vote insincerely". In a voting game with a finite number of voters,

\footnotetext{
${ }^{1}$ Its detractors claim that this rule enhances strategic voting when compared for instance to $P V$, whereas its proponents consider that it has several advantages as far as strategic voting is concerned. For an extensive discussion of this controversy, the reader can refer to Brams [8], Brams and Fishburn [9], and Weber [39].

${ }^{2}$ Few papers deal with the properties of these games: see Ahn and Oliveros [1], Bouton and Castanheira [7], Goertz and Maniquet [18], De Sinopoli and Gonzalez-Pimienta [11], Krishna and Morgan [19], Núñez[32, 33].
} 
de Sinopoli, Dutta and Laslier [10] actually prove that "for every pure strategy of the other players, the set of best replies contains a sincere strategy" whereas this is no longer true when one considers mixed strategies ${ }^{3}$.

The main difference between these examples is the source of insincerity. Whereas in Example 1 voters do not approve of a candidate as they expect him to have no votes, in Example 2 their insincerity is a consequence of the Poisson structure of the game. This difference sheds some light on the properties of Poisson games.

If a candidate gets no votes, then the voter skips him depending on her intensity of preference. In Example 1, the voter prefers the lottery between her first and third candidate to the victory of her second preferred candidate. Hence, she skips her second preferred candidate in equilibrium. This sort of insincerity can be removed since it is based on the cardinal utilities of the voters. Indeed, assume that the voter prefers the victory of some candidate $i$ over the victory of any other set of candidates, at least one of them being less preferred than $i$. This candidate is denoted as strong. We prove that no voter's best response skips a strong candidate provided that this candidate has no votes. Note that a candidate ranked first by a voter is always strong as the voter prefers the victory of this candidate to any other outcome in the election. Hence, whenever the set of top candidates (assuming strict preferences, all candidates but the two last ranked ones) equals the set of strong candidates, there is no equilibrium in which no-skipping sincerity is violated on the mere reason of a candidate having no votes.

If a candidate gets a positive share of the votes, then the incentives for (in)sincerity become very different in a Poisson game. Indeed, with positive probability, this candidate might win the election. This fact combined with the correlation of the scores of the candidates that arise in these games is enough to prove a surprising result. Take a voter with preferences $1>2>3>4$ and assume that 1 is the winner in equilibrium. We prove that it might be a strict best response to approve of 1 and 3 in equilibrium. Indeed, the most likely pivot outcome involving 2 is against 1 whereas the most probable pivotal event in which 3 is present involves both 1 and 4 . Hence, approving of candidate 2 does not depend on the voter' intensity of preferences towards him whereas she approves of 3 as long as the utility of 3 is high enough. We include a formal discussion on how this result hinges on the Poisson structure; indeed if one assumes that the scores of the candidates are independent random

\footnotetext{
${ }^{3}$ We can hence conclude that the strategic behavior in Poisson games is related to the one in normal-form games when voters use mixed-strategies. Moreover, in a related context, Laslier [21] proves that, under some general conditions, no-skipping sincerity is ensured in a large election model. The differences between such a setting and ours are discussed in Section 5.
} 
variables, this sort of phenomena should not arise.

Finally, we set up a comparison of our results with the strategic behavior under rank scoring rules. It is possible to prove by a simple extension of Myerson [28] that the rest of scoring rules fail to satisfy no-skipping sincerity. More interestingly, we identify two intuitive weak versions of sincerity that prove that the sincere behavior of $A V$ arises as it is a combination of Negative voting and Plurality Voting. Indeed, we prove that Plurality voting is the unique rank scoring rule under which a voter might give zero points to her most preferred candidate. At the same time, it is also the only one of these rules that prevents a rational voter to give a positive score to her least preferred candidate. Similarly, Negative voting captures some strong notion of sincerity as it is the only positional rule that ensures that in equilibrium a voter assigns a score of 1 to her most preferred candidate.

This paper is structured as follows. Section 2 presents a review of the literature and Section 3 introduces the basic model. Sections 4 and 5 focus on the strategic sincerity of $A V$ and provide the sufficient conditions for sincerity; Section 6 presents the results on rank scoring rules and Section 7 concludes.

\section{Sincerity as a benchmark}

Up to now, we have not addressed the question of why one should focus on the sincerity of $A V$. First of all, note that, according to the previous literature in strategic voting, it is clear that the rest of scoring rules do not trigger no-skipping sincerity in equilibrium (see for instance the examples by Myerson [28]). In other words, as $A V$ is the unique scoring rule that ensures no-skipping sincerity with three candidates. It hence seems important to understand sincerity in environments with more candidates.

Moreover, the concept of sincerity is related, even though, not equivalent to the strategy-proofness condition used in the well-known impossibility theorems from the 70s. A paper closely related to ours is the extension of the Gibbard-Satterthwaite theorem to a Poisson environment as proposed by McLennan [23]. McLennan [23] proves that any decision scheme $e^{4}$ that satisfies some reasonable conditions (in the spirit of strategy-proofness and unanimity) is a random dictatorship in Poisson games with at least three candidates ${ }^{5}$. The key difference between such a result

\footnotetext{
${ }^{4}$ In a decision scheme, voters announce a preference profile and given such an announcement the outcome of the scheme is a lottery over the different candidates.

${ }^{5}$ Our contribution is also related to the literature on the vulnerability of other types of mechanism
} 
and the sincerity of $A V$ with three candidates is that under $A V$ there are different sincere messages. Indeed, if a voter prefers candidate 1 to candidate 2 to candidate 3 and votes for the two first ones, it may be the case that she votes as if she preferred candidate 2 to candidate 1 to candidate 3 . Hence, the same message is considered as sincere for two different preference profiles. Note that under $P V$ or the Borda rule, the set of sincere messages is unique given the voter's preferences ${ }^{6}$. This is why the literature has proposed several definitions of sincerity under $A V$ whereas the meaning of sincerity in a classical social choice theory model is unambiguous: a voter sincerely communicates her type to the social planner. In other words, as argued by Sanver [37] and Endriss [14], $A V$ does not fit in the canonical model of voting studied in social choice theory.

One of the main reasons used to advocate sincerity in social choice theory, as pointed by Dowding and Van Hees [13], is that insincere behavior is unfair since it provides an advantage to smarter voters. These voters may indeed be able, through sophisticated insincere behavior, to manipulate the outcome of the election in their favor. Such a feature may be particularly undesirable in a democratic situation since all voters are supposed to have the same voting rights. Yet another reason would be that behaving sincerely is less costly for voters than behaving insincerely. Voting sincerely for your preferred candidate(s) seems indeed less effort intensive than choosing an optimal strategic behavior in order to manipulate the outcome in one's favor. Such low "thinking" costs would therefore be an argument in favor of an electoral system since it would be less costly to use than other systems in which voters have to think much more carefully about their voting behavior. Note that such an argument needs not apply to approval voting since there are many different sincere ballots, which makes the decision harder for the voters.

Finally, if one accepts that sincerity is a good benchmark to evaluate electoral systems $^{7}$, it is not obvious which one among the specific definitions provided in

to manipulation. For instance, Pathak and Sonmez [36] study a notion of degree of manipulation in the college admissions problem (see Barbera [3] for a recent survey on the concept of strategyproofness).

${ }^{6}$ See Wolitzky [40] for a discussion on the relation between strategy-proofness and sincerity in voting.

${ }^{7}$ Note that when voters are sincere, $A V$ does not ensure reasonable preference aggregation as shown by Núñez [33] in Poisson games and De Sinopoli et al. [10] with traditional equilibrium refinements such as stable sets. Such an intuition is also present in information-aggregation models. In a different setup, the literature on the Condorcet Jury Theorem (Feddersen and Pesendorfer [15, $16,17]$ and Myerson [25]) suggests that insincere voting may actually lead to a better social outcome than sincere voting. Indeed, when voters are not sincere the best candidate is the only likely winner even if information is biased. This may not the case when voters are sincere. 
the literature is the appropriate one. We hence consider all of them. As already mentioned, no-skipping sincerity follows from Brams and Fishburn (1978) in their definition of a sincere ballot. This definition is intuitive and broadly accepted. Nonetheless, the other definitions of sincerity present in Merrill and Nagel [24] are of independent interest. Recently, Dowding and Van Hees [13] provide a definition of sincere manipulation, building on the idea that one should not consider all manipulations alike. They prove that a wide class of voting rules to which $P V$ belongs is immune to an undesirable type of manipulation. We do not evaluate their definition of sincerity in the current framework as their main definition of sincere manipulation is defined in terms of groups of voters and hence a natural adaptation in a context with a random number of voters is far from obvious.

\section{Poisson Voting Games}

Each voter has a type $t$ in set of types $T$ that defines her strict cardinal preferences over the set of candidates $K=\{1,2, \ldots, k\}$. A voter's payoff only depends on the candidate who is elected. The preferences of a voter with a type $t$ are denoted by $u_{t}=$ $\left(u_{t}(k)\right)_{k \in K}$. Preferences are assumed to be strict. Each voter's type is independently drawn from $T$ according to the distribution of types denoted by $r=(r(t))_{t \in T}{ }^{8}$. In other words, $r(t)$ represents the probability that a voter randomly drawn from the population has type $t$.

A Poisson voting Game of expected size $n$ is a game such that the actual number of voters taking part in the election is a random variable drawn from a Poisson distribution with mean $n^{9}$. The probability distribution and its parameter $n$ are common knowledge. A finite Poisson game of expected size $n$ is then represented by $(K, T, n, r, u)$. The expression "Large Poisson game" or LPG refers to the asymptotic behavior of a sequence of Poisson games of expected size $n$ when $n$ is large enough.

In order to completely determine an election in a Poisson voting game, the voting rule remains to be specified. The set of available ballots is denoted by $C$. Formally, each ballot is a vector $c$ of length $k$. Under $A V$, each coordinate $c_{i}$ being equal to 0 or to 1 . If $c_{i}=1$, candidate $i$ is approved by ballot $c$. We let $Z(C)$ denote the set of possible action profiles for the players in a Poisson game. That is, $Z(C)$ is the set of

\footnotetext{
${ }^{8}$ The distribution of types satisfies $r(t) \geq 0 \forall t \in T$ and $\sum_{t \in T} r(t)=1$.

${ }^{9}$ A Poisson random variable $\mathcal{P}(n)$ is a discrete probability distribution that depends on a unique parameter (its mean). The probability that a Poisson random variable of parameter $n$ takes the value $l$, being $l$ a nonnegative integer equals $e^{-n} \frac{n^{l}}{l !}$.
} 
vectors $x=(x(c))_{c \in C}$ such that each component $x(c)$ is a nonnegative integer.

As shown by Myerson [25], voters' actions uniquely depend on their private information $t$ on this type of games in equilibrium (common public information). Moreover, in these games the number of voters who choose a given ballot is independent from the number of voters who choose another ballot (independence of actions).

We represent voters' actions by the strategy function $\sigma(c \mid t){ }^{10}$ which is a function from $T$ into $\Delta(C)$ the set of probability distributions over $C$. A voter with type $t$ chooses ballot $c$ with probability $\sigma(c \mid t)$. Then, given the distribution of types $r$ and the strategy function $\sigma(. \mid t)$, the vote distribution $\tau=(\tau(c))_{c \in C}$ can be determined as follows. For each $c \in C$, we define $\tau(c)=\sum_{t \in T} r(t) \sigma(c \mid t)$.

The vote distribution $\tau$ represents the share of votes each ballot gets. Given the vote distribution $\tau$, the (common knowledge) probability that the outcome is equal to a vector $x \in Z(C)$ is such that

$$
P[x \mid n \tau]=\prod_{c \in C}\left(\frac{e^{-n x(c)}(n \tau(c))^{x(c)}}{x(c) !}\right) .
$$

Under $A V$, we let $\mathcal{C}_{j}$ denote the set of ballots in which candidate $j$ is approved. Given the vote distribution $\tau$, the expected score distribution $\rho=(\rho(j))_{j \in K}$ describes the expected number of points each candidate gets. For each $j \in K$,

$$
\rho(j)=\sum_{c \in \mathcal{C}_{j}} \tau(c) c_{j}
$$

The candidates with the highest score $\rho(\cdot)$ are the front-runners of the election. The random variable that describes the number of votes each candidate $j$ gets is denoted $s(j)$ and it is a Poisson variable of parameter $n \rho(j)$.

For each event $x \in Z(C)$, the actual scores of the candidates are described by the vector $s_{x}=\left(s_{x}(j)\right)_{j \in K}$ with $s_{x}(j)$ with $s_{x}(j)=\sum_{c \in \mathcal{C}_{j}} x(c)$. When the vote profile is $x \in Z(C)$, let $M(x)=\arg \max _{j \in K} s_{x}(j)$ denote the set of candidates with the highest number of points. Assuming a fair toss of a coin, the probability of candidate $k$ winning the election given the vector $x \in Z(C)$ is

\footnotetext{
${ }^{10}$ The strategy function satisfies $\sigma(c \mid t) \geq 0 \forall c \in C$ and $\sum_{d \in C} \sigma(d \mid t)=1$. It plays the role of a strategy combination in a game with a constant number of players.
} 


$$
W[k \mid x]= \begin{cases}1 / \#(M(x)) & \text { if } k \in M(x) \\ 0 & \text { if } k \notin M(x) .\end{cases}
$$

For any vector $x \in Z(C)$ and any ballot $c \in C$, we let $x+\{c\}$ denote the vector such that one ballot $c$ is added. Thus, given the vote profile $x$, a voter with type $t$ casts the ballot $c$ that maximizes her expected utility

$$
U_{t}[c]=\sum_{x \in Z(C)} P[x \mid n \tau] \sum_{k \in K} W[k \mid x+\{c\}] u_{t}(k) .
$$

Definition 1. We refer to $\sigma$ as an equilibrium of a finite Poisson voting game if for each $c \in C$ and each $t \in T$,

$$
\sigma(c \mid t)>0 \Longrightarrow c \in \arg \max _{d \in C} U_{t}[d]
$$

Nevertheless, as the focus of this work is on elections with a large number of voters, one shall look at the limits of equilibria as the expected number of voters $n$ tends to infinity. Thus, we refer to a large equilibrium sequence to denote any equilibrium sequence $\left\{\sigma_{n}\right\}_{n \rightarrow \infty}$ of the finite voting games of expected size $n$ such that the vectors $\sigma_{n}$ are convergent to some limit $\sigma$ as $n \rightarrow \infty$ in the sequence. We refer to this limit $\sigma$ as a large equilibrium. Furthermore, we refer to a sequence of outcomes in $Z(C)$ by $\left\{x_{n}\right\}_{n \rightarrow \infty}$. The limit $x$ of a sequence of vectors $\left\{x_{n}\right\}_{n \rightarrow \infty}$ in $Z(C)$ is a vector of $Z(C)$.

We assume that each voter determines which ballot he casts by maximizing her expected utility: a voter cares only about the winner's identity. As we analyze elections with a large number of voters, a voter's action has no impact in almost any possible outcome of the election. Indeed, it has some impact if and only if there is some set of candidates involved in a close race for first place where one ballot pivotally changes the result of the election: a pivot ${ }^{11}$.

For some ballot $c$ and a pair of candidates $i$ and $j$, pivot $(c, i, j)$ denotes the event that adding one more ballot $c$ can change the winner from candidate $i$ to candidate $j$,

$$
\operatorname{pivot}(c, i, j)=\{x \in Z(C) \mid W[i \mid x]>W[i \mid x+\{c\}] \text { and } W[j \mid x]<W[j \mid x+\{c\}]\}
$$

Let pivot $(i, j)$ denote the event in which there is a close race such that one additional vote can pivotally change the winner of the election from one to the other of

\footnotetext{
${ }^{11} \mathrm{~A}$ brief discussion of the methods to compute such probabilities is included in the appendix. The interested reader might find the insights presented by Myerson [27], Myerson [28], Núñez [32] and Núñez [33].
} 
these two candidates,

$$
\operatorname{pivot}(i, j)=\bigcup_{c \in C}(\operatorname{pivot}(c, i, j) \cup \operatorname{pivot}(c, j, i)) .
$$

The event pivot $(i, j)$ is the union of the different outcomes in which one single ballot can change the outcome of the election from one to the other.

Voters take into account only the probabilities of these events in order to determine their best responses. We will often refer to the magnitude of an outcome rather than to its probability, as it simplifies the computations. An outcome $A$ is a subset of $Z(C)$ with $P[A \mid n \tau]=\sum_{x \in A} P[x \mid n \tau]$. Given a large equilibrium sequence $\left\{\sigma_{n}\right\}_{n \rightarrow \infty}$, the magnitude $\mu[A]$ of an outcome $A$ is such that

$$
\mu[A]=\lim _{n \rightarrow \infty} \frac{1}{n} \log P[A \mid n \tau] .
$$

A description of the computational techniques is included in the appendix.

For any vote profile $x$, any expected vote profile $n \tau$, and any ballot $c$, we denote the limit of the $c$-offset ratios by

$$
\alpha(c)=\lim _{n \rightarrow \infty} \frac{x(c)}{n \tau(c)} .
$$

The offset theorem (Myerson [27]) states that, given $n \tau$, for any outcome $A \in Z(C)$ and any vector $w \in Z(C)$,

$$
\lim _{n \rightarrow \infty} \frac{P[A-w \mid n \tau]}{P[A \mid n \tau]}=\prod_{c \in C} \alpha(c)^{w(c)} .
$$

\section{Skipping a candidate with no votes: Intensity of Pref- erences}

Whereas the meaning of sincerity is clear in a rule like $P V$ (a sincere voter simply votes for her preferred candidate), it is not that simple in a rule like $A V$ where the voter can vote for different candidates. Indeed, there has been a controversy over this point ${ }^{12}$.

Merril and Nagel [24] present the different notions of sincerity under Approval voting considered in the literature, included here for completeness.

\footnotetext{
${ }^{12}$ For a summary of the properties of $A V$, see the recent book by Laslier and Sanver [22].
} 
- No-Skipping: A voter approves of all the candidates preferred to the least preferred approved candidate.

- Pure Sincerity: A voter approves of all the candidates whose utility is above the average utility without skipping.

- Expansive Sincerity: A voter votes for more than the candidates prescribed by pure sincerity without skipping.

- Restrictive Sincerity: A voter votes for less than the candidates prescribed by pure sincerity without skipping.

- Weak Insincerity: A voter may skip some candidates but votes for her preferred choice.

- Strong Insincerity: A voter may skip some candidates and does not vote for her preferred choice.

The no-skipping notion was proposed by Brams and Fishburn (1983) whereas the pure sincerity was suggested by Merril and Nagel [24], in which a discussion of the motivations of the different versions of sincerity is provided. More recently, Ballester and Rey-Biel [2] analyze sincerity under voting rules in a mechanism design fashion, in the absence of information over the other voters' preferences. None of the previous works are performed in a game-theoretical framework, in contrast with ours. Our approach assumes complete information over the other voters' preferences even though the Poisson distribution creates an uncertainty over the size of the population.

For precision, we include a formal definition of both no-skipping sincerity and pure sincerity as they will be the main focus of the current work.

Definition 1: An $A V$ ballot satisfies no-skipping sincerity for a $t$-voter if $u_{t}(i)>$ $u_{t}(j) \Longrightarrow c_{i} \geq c_{j}$.

Definition 2: An $A V$ ballot satisfies pure sincerity for a $t$-voter if satisfies noskipping sincerity and $u_{t}(i)>\frac{\sum_{j \in K} u_{t}(j)}{k} \Longleftrightarrow c_{i}>0$.

Pure Sincerity: Note that the notion of pure sincerity seems poorly adapted to a strategic environment. Furthermore, it suffices to check the equilibrium depicted by Núñez [33] in order to see that pure sincerity does not hold under $A V$, as in order to construct the equilibrium, one only needs to specify the ordinal preferences ${ }^{13}$. Both

\footnotetext{
${ }^{13}$ I would like to thank one referee for pointing this out.
} 
expanded and restricted sincerity closely follow the idea of pure sincerity and are based on no-skipping sincerity.

As will be shown, we prove that in general voters might skip a candidate in equilibrium. More specifically, we prove that there exist equilibria in which a voter's unique best response is to skip a candidate. Notwithstanding, we differentiate between two main reasons for skipping a candidate.

The first one arises when every voter assumes that some candidate $l$ receives no votes at all. In this situation, it seems intuitive to think that the voter is indifferent between approving or not candidate $l$. As will be proved, it might lead a strictly higher expected payoff not to approve it. Nonetheless, we prove that under a suitable restriction of the voter's intensities, this sort of skipping does not arise in equilibrium.

The second reason seems more deeply rooted in the Poisson structure on the game. Indeed, we provide an example in which a candidate has a positive share of votes (an expected $1 / 8$ of the total number of votes) and some voters rationally skip him. This is due to the correlations between the scores of the candidates that arise in the equilibrium described. A discussion on how a different model would account for such sort of insincerity is presented.

\subsection{An example}

The next example proves that the voters need not approve of a candidate when they anticipate that this candidate receives no vote.

Take a LPG with $K=\{1,2,3,4\}$ and in which voters' utilities are as follows:

$$
u_{t_{1}}=(10, s, r, 0) ; u_{t_{2}}=(1,3,10,0) \text { and } u_{t_{3}}=(0,1,3,10) \text {, }
$$

with $10>s>r>0$. Besides the distribution of types satisfies

$$
0<r\left(t_{1}\right)<r\left(t_{2}\right)<r\left(t_{3}\right)<\frac{1}{2} .
$$

Remark: Voters' preferences in this situation are consistent with single-peakedness. It is not difficult to see that $t_{1}$-voters' best responses are not sincere with the strategy function $\sigma_{1}$ depicted as follows:

$$
\sigma_{1}=(\{1,3\},\{3\},\{4\}),
$$


in which $t_{1}$-voters vote $\{1,3\}, t_{2}$-voters vote $\{3\}$ and $t_{3}$-voters approve of just candidate 4 .

We show now that $\sigma_{1}$ describes a strict large equilibrium as long as $s<\frac{10+r}{3}$. The intuition for this condition over the voters' intensities is as follows. Indeed, given $\sigma_{1}$, candidate 2 gets no votes. Hence, there are only two events in which a $t_{1}$-voter is not indifferent between casting ballot $\{1,3\}$ (as in $\sigma_{1}$ ) or to add candidate 2 to her ballot (to vote $\{1,2,3\}$ ): either every voter abstains (with probability $e^{-n}$ ) or only one $t_{3}$-voter takes part in the election (with probability $n r\left(t_{3}\right) e^{-n}$ ).

As we deal with large elections, we focus on the limit of these probabilities when $n$ tends towards infinity. We let $A=(0,0,0,1)$ and $w=(0,0,0,1)$ so that $A$ is the event in which only one $t_{3}$-voter takes part in the election whereas $A-w$ is the one in which every voter abstains. Hence, the offset theorem (introduced in Section 3) entails that

$$
\lim _{n \rightarrow \infty} \frac{P[A-w \mid n \tau]}{P[A \mid n \tau]}=\alpha(4),
$$

with $\alpha(4)=\lim _{n \rightarrow \infty} \frac{1}{n r\left(t_{3}\right)}=0$. Therefore, when $n$ is large enough, a $t_{1}$-voter only takes into account the event $A$. In such an event, if he votes $\{1,2,3\}$, the outcome is a lottery between all the candidates hence her utility equals $\frac{10+r+s}{4}$. On the contrary, if he votes $\{1,3\}$, the lottery takes place between candidates 1,3 and 4 and hence her utility equals $\frac{10+r}{3}$. Therefore, he prefers to vote $\{1,3\}$ as long as $\frac{10+r+s}{4}<\frac{10+r}{3}$, which is equivalent to $s<\frac{10+r}{3}$. Similarly, $t_{2}$ and $t_{3}$ voters do not vote for 2 as with the participation of at most one voter, it is never in their interest to approve of this candidate.

The approvals of candidates 1,3 and 4 by the different voters remain to be clarified; as they depend on the ordering of the pivot outcomes, we divide the discussion by the different pivot outcomes in which each of the candidates is included.

\section{Pivots in which 1 is involved.}

The $t_{1}$ voters have 1 as their most preferred candidate, so that they approve of 1 . Similarly, the $t_{3}$ voters have 1 as their least preferred candidate so that they do not vote for 1 . Finally, given $\sigma_{1}$, the voters who vote for 1 vote also for 3 . It follows that in any pivot outcome in which 1 is involved, so is 3 . Hence, as the $t_{2}$ voters prefer 3 to 1 they do not approve of 1 .

\section{Pivots in which 3 is involved.}

The probability of pivot $(3,4)$ equals the one of the event $\{x(1,3)+x(3)=x(4)\}$ whereas the probability of pivot $(1,3)$ is equivalent to the probability of $\{x(3)=0\} \cap$ 
$\{x(1,3) \geq x(4)\}$. The magnitude of pivot $(3,4)$ equals $-\left(\sqrt{r\left(t_{1}\right)+r\left(t_{2}\right)}-\sqrt{r\left(t_{3}\right)}\right)^{2}$ which is higher than the one of pivot $(1,3)\left(-r\left(t_{2}\right)-\left(\sqrt{r\left(t_{1}\right)}-\sqrt{r\left(t_{3}\right)}\right)^{2}\right)$. Hence, the voters who prefer 3 to 4 approve of 3 ( $t_{1}$ and $t_{2}$ voters) and the ones who prefer 4 to 3 do not ( $t_{3}$ voters).

\section{Pivots in which 4 is involved.}

Given the voters' best responses, the probability of pivot $(3,4)$ equals the one of the event $\{x(1,3)+x(3)=x(4)\}$ whereas the probability of pivot $(1,4)$ is equivalent to the probability of $\{x(3)=0\} \cap\{x(1,3)=x(4)\}$. It hence follows that pivot $(3,4)$ has the highest magnitude implying that the voters who prefer 4 to 3 approve of 4 ( $t_{3}$ voters) and the ones who prefer 3 to 4 do not ( $t_{1}$ and $t_{2}$ voters).

We have therefore proved the following proposition.

Proposition 1. Whenever the utility of candidate 4 is lower than the average utility for $t_{1}$-voters $\left(s<\frac{10+r}{3}\right)$, the strategy function $\sigma_{1}$ is a strict large equilibrium. In this equilibrium, skipping is a strict best response for $t_{1}$-voters.

As a consequence of Proposition 1 we can establish an important consequence concerning strategic behavior under $A V$ in Poisson Games.

Corollary 1. There exists equilibria in which skipping a candidate is a strict best response.

Corollary 2. There exists equilibria in which violating expanded/restricted/pure sincerity is a strict best response.

\subsection{A condition for approving of a Candidate with No Votes}

Let us recall that the voters are assumed to have strict preferences over the candidates. For the remaining of this section, we assume that the $t$-voter's preferences are $u_{t}(1)>u_{t}(2)>\ldots>u_{t}(k)$ for ease of exposition.

For any candidate $i$ in $K$, we define the upper-contour set of $i$ for $t$-voters by the set of candidates strictly preferred by $t$-voters to $i$. Formally, we denote it by:

$$
\operatorname{UCS}_{t}(i)=\left\{j \in K \mid u_{t}(j)>u_{t}(i)\right\}
$$

Given the uniform tie-breaking rule, one can derive preferences over the different winning sets (that is over subsets of the set of candidates) from the preferences over the candidates. Indeed, for any pair of subsets of candidates $X, Y \subseteq K$, we write 
$X \geq_{t} Y$ to denote that the $t$-voter (weakly) prefers the lottery over the candidates in $X$ rather than the lottery over the candidates in $Y$. Due to the uniform tie-breaking rule, it is equivalent to write that

$$
X \geq_{t} Y \Longleftrightarrow \frac{1}{|X|} \sum_{x \in X} u_{t}(x) \geq \frac{1}{|Y|} \sum_{y \in Y} u_{t}(y),
$$

where |.| stands for the cardinal. The relation $\geq_{t}$ defines a weak order over the different sets of candidates.

Candidate $i$ is a strong candidate for type- $t$ voters if they always prefer to add candidate $i$ to any lottery not included in the upper-contour set of $i$. In other words, candidate $i$ is strong for type- $t$ voters if and only if for any $Y \nsubseteq U C S_{t}(i)$,

$$
Y \cup\{i\}>_{t} Y \Longleftrightarrow\{i\}>_{t} Y,
$$

with $Y \cup\{i\} \neq Y$. We denote the set of such candidates by $\mathcal{S}_{t}(K)$.

The next theorem states there cannot exist an equilibrium in which a voter skips a strong candidate. This result characterizes an important and distinctive feature of Approval Voting. Indeed, as proved by Myerson [28], the Plurality rule (voting for one candidate) is too much vulnerable to voters' anticipations. As is well-known, the wasted-vote effect is recurrently associated to Plurality rule. Whenever the voters anticipate that a candidate receives no votes, no voter votes for him since it is becomes rationally dominated. Hence, the voters have a tendency to vote for their most preferred candidate among the ones with a positive probability of winning. One of the main arguments for Approval Voting is that this wasted-vote effect is less severe. A good example of this feature is to take a society with a unanimously preferred candidate. Under $A V$, this candidate is the unique winner since every one votes for her most preferred candidate whereas it need not be the case under $P V$. Theorem 2 reinforces this feature since it proves that under $A V$ voters need not skip candidates on the sole reason of the candidate having no votes as it is the case under $P V$.

Theorem 1. Let $k \geq 4$. There is no equilibrium in which a voter skips a strong candidate with no votes.

Proof. Assume that the $t$-voter's preferences are $u_{t}(1)>u_{t}(2)>\ldots>u_{t}(k)$. The argument is first done for the case in which $k=4$ for simplicity of exposition.

\section{4 candidates}


We assume that 2 is a strong candidate. Let $\sigma$ be an equilibrium in which the $t$-voters vote $\{1,3\}$ and so skip 2 . Assume moreover that no other voter approves of candidate 2. It follows that the only outcomes $x \in Z(C)$ in which voting for 2 is pivotal are the ones in which each candidate but 2 gets at most one point: the events in the set $X_{2}=\left\{x \in Z(C) \mid s_{x}=\left(s_{x}(j)\right)_{j \in K}\right.$ with $s_{x}(2)=0$ and $\left.s_{x}(j) \in\{0,1\}\right\}$.

Consider the decision of the voters between ballot $\{1,3\}$ and ballot $\{1,2,3\}$ at the different events $x \in X_{2}$. Let us recall that $M(x)$ stands for the set of candidates with highest score at each profile $x$. As no voter votes for 2 , there is only one event $x \in X_{2}$ with $2 \in M(x)$ in which every voter abstains and hence $M(x)=\{1,2,3,4\}$.

- If $M(x)=\{4\}$, then $M(x+\{1,2,3\})=\{1,2,3,4\}>_{t}\{1,3,4\}=M(x+\{1,3\})$.

- If $M(x)=\{1,2,3,4\}$, then $M(x+\{1,2,3\})=\{1,2,3\}>_{t}\{1,3\}=M(x+\{1,3\})$.

- If either 1 or 3 are in $M(x)$ with $M(x) \neq K$, then $M(x+\{1,2,3\}) \sim_{t} M(x+\{1,3\})$. Indeed, adding a point to a candidate already in the winning set ensures that he still belongs to it and both ballots assign one point to both 1 and 3 .

Therefore, the $t$-voter strictly prefers to vote $\{1,2,3\}$ since the probability of every voter abstaining is strictly positive which implies that $\sigma$ is not an equilibrium.

\section{$\mathrm{k}$ candidates}

Let $\sigma$ be an equilibrium in which some $t$-voter votes for some subset of candidates $A \subseteq K$. We denote by $K \backslash A$ the set of non-approved candidates. Assume that the voter skips some candidate $m$ so that he approves of some candidate $n$ with $u_{t}(m)>u_{t}(n)$ with $n \in A$ and $m \in K \backslash A$.

Take any skipped candidate $m$ and assume it is strong: it follows that $A \cup\{m\}>_{t} A$ since $A \not \subset U C S_{t}(m)$ since $n \in A$ and $u_{t}(m)>u_{t}(n)$.

Assume that no voter approves of candidate $m$ according to $\sigma$. It follows that the only outcomes $x \in Z(C)$ in which voting for $m$ is pivotal are the ones in which each candidate but $m$ gets at most one point: the events in the set

$$
X_{m}=\left\{x \in Z(C) \mid s_{x}=\left(s_{x}(j)\right)_{j \in K} \text { with } s_{x}(m)=0 \text { and } s_{x}(j) \in\{0,1\}\right\}
$$

$M(x)$ and $M(x+B)$ respectively stand for the candidates with the most votes given $x$ and $x+B$ for any ballot $B \subseteq K$. Moreover, since under $A V$, a ballot $B$ can be simply expressed as a subset of the candidates note that $M(B)=B$. As for each $x \in X_{m}$, each candidate has a score of at most one, the following simple rule determines the set of winners for any $x+B$ : 
- if $M(x) \cap M(B) \neq \emptyset$, then $M(x+B)=M(x) \cap M(B)$,

- if $M(x) \cap M(B)=\emptyset$, then $M(x+B)=M(x) \cup M(B)$.

Consider the decision of the voters between ballot $A$ and ballot $A \cup\{m\}$ at the different events $x \in X_{2}$.

- If $M(x)=K$, then $M(A), M(A \cup\{m\}) \subset M(x)$ so that $M(x) \cap M(A), M(x) \cap M(A \cup$ $\{m\}) \neq \emptyset$. Therefore, $M(x+A \cup\{m\})=A \cup\{m\}>_{t} A=M(x+A)$.

- If $M(x) \subseteq A$, then $M(x) \subseteq M(A)$ since $M(A)=A$ by definition. Hence, we have that $M(x) \cap M(A) \neq \emptyset$, so that $M(x+A)=M(x) \cap M(A)=M(x)$. Similarly, as $M(x) \subseteq$ $A \subset A \cup\{m\}$, it follows that $M(x+A \cup\{m\})=M(x)$. Hence, $M(x+A \cup\{m\}) \sim_{t} M(x+A)$.

- If $M(x) \subseteq K \backslash\{A-\{m\}\}$, then $M(x) \cap M(A)=\emptyset$. Then, $M(x+A)=M(x) \cup M(A)=$ $M(x) \cup A$. Similarly, $M(x) \cap M(A \cup\{m\})=\emptyset$ so that $M(x+A \cup\{m\})=M(x) \cup A \cup\{m\}$. Since $M(x) \cup A \not \subset \cup C S_{t}(m)$, it follows that $M(x) \cup A \cup\{m\}>_{t} M(x) \cup A$ and hence $M(x+A \cup\{m\})>_{t} M(x+A)$.

- If $M(x) \nsubseteq A$ and $M(x) \nsubseteq K \backslash\{A-\{m\}\}$, then $M(x) \not \subset U C S_{t}(m)$. Moreover, $M(x) \cap$ $M(A) \neq \emptyset$ (otherwise $M(x) \subseteq K \backslash\{A-\{m\}\})$ so that $M(x+A)=M(x) \cap M(A)=M(x) \cap A$. Similarly, $M(x) \cap M(A \cup\{m\}) \neq \emptyset$ so that $M(x+A \cup\{m\})=M(x) \cap(A \cup\{m\})=M(x) \cap A$ since $m \notin M(x)$. Therefore $M(x+A \cup\{m\}) \sim{ }_{t} M(x+A)$.

Therefore, the $t$-voter strictly prefers to vote $A \cup\{m\}$ proving that $\sigma$ is not an equilibrium.

Building on the previous results, we can set up a very simple condition to ensure that a voter with a given type applies no-skipping sincerity.

We denote the set of top candidates for a $t$-voter denotes all candidates but the two last ranked ones:

$$
\mathcal{T}_{t}(K)=\{1, \ldots, k-2\} .
$$

The reason why we need to state the condition in terms of top candidates (all candidates but the two last ranked ones) is intuitive. Note that the result does not state which set of candidates is approved. Hence, in order to be sure that, independently of the set of approved candidates, the voter's best response satisfies no-skipping sincerity, we must ensure that any candidate above the lowest possible candidate that can be approved is strong. As a strategic voter never approves her lowest preferred 
candidate, the lowest ranked candidate he might approve is the candidate ranked last but one. Hence, all candidates ranked above the last but one must be strong in order to ensure no-skipping sincerity.

Corollary 3. Let $k \geq 4$. If for some $t \in T, \mathcal{T}_{t}(K)=\mathcal{S}_{t}(K)$, there is no equilibrium in which the $t$-voters do not approve of a strong candidate with no votes.

Proof. Let $\mathcal{T}_{t}(K)=\mathcal{S}_{t}(K)$. The fact that no-skipping sincerity is ensured is a simple consequence of Theorem 1 whenever $k \geq 4$, as any strong candidate is approved, provided that a lower ranked candidate is approved of by the strategic voter.

The previous condition on the voter's preferences (namely the set of top candidates equals the set of strong candidates) is highly implicit ${ }^{14}$. Indeed, it is not easy to check whether the condition holds as one needs to determine the individual's utility over each of the different sets of candidates not included in the upper contour set of each top candidate. We now provide a characterization of this condition that greatly simplifies this task as one simply needs to check one inequality per candidate to determine whether the condition for sincerity holds. It turns out that with four candidates, assuming that the utility over the second ranked candidate is above the utility of the lottery between the first and the third ranked candidate of some type of voters is equivalent to assume that the set of top candidates equals the set of strong candidates. With any number of candidates, one needs to require for every top candidate $i \in T_{t}(K)$ that the voter prefers the victory of $i$ to the tie between all the candidates ranked above $i$ and the candidate $i+1$, ranked immediately below $i$.

Proposition 2. Let $k \geq 4$. For some $t \in T$,

$$
\mathcal{T}_{t}(K)=\mathcal{S}_{t}(K) \Longleftrightarrow\{i\}>_{t}\{1, \ldots, i-1\} \cup\{i+1\},
$$

for every $i \in \mathcal{T}_{t}(K)$.

Proof. $\Rightarrow)$ Assume first that for some $t \in T, \mathcal{T}_{t}(K)=\mathcal{S}_{t}(K)$ with $u_{t}(1)>u_{t}(2)>\ldots>$ $u_{t}(k)$. Take any candidate $i \in \mathcal{T}_{t}(K)$. It follows that $i \in \mathcal{S}_{t}(K)$ so that $Y \cup\{i\}>_{t} Y$, for any $Y \nsubseteq U C S_{t}(i)$. Then, letting $Y=\{1, \ldots, i-1\} \cup\{i+1\}$, it follows that $\{1, \ldots, i-1, i, i+$ $1\}>_{t}\{1, \ldots, i-i, i+1\}$, which is equivalent to $\{i\}>_{t}\{1, \ldots, i-1, i+1\}$ as required.

$\Leftarrow)$ Assume now that $\{i\}>_{t}\{1, \ldots, i-1\} \cup\{i+1\}$, for every $i \in \mathcal{T}_{t}(K)$. Let us now prove that each $i \in \mathcal{I}_{t}(K)$ is strong so that $Y \cup\{i\}>_{t} Y$, for any $Y \nsubseteq U C S_{t}(i)$ and $i \notin Y$.

\footnotetext{
${ }^{14}$ I would like to thank a referee for pointing this out
} 
The proof proceeds by induction on the candidates.

a. Candidate 1. The claim is immediate as candidate 1 is strong independently of the voters' preferences.

b. Candidate 2. Take first any set $Y$ of candidates with $\#(Y)=1$. By definition, $\{2\}>_{t}\{i\}$ for any $i \in\{3, \ldots, k\}$ which implies that $Y \cup\{i\}>_{t} Y$ as long as $\#(Y)=1$. Consider now any set $Y$ with $\#(Y)=2$. By assumption, $\{2\}>_{t}\{1,3\}$. Moreover, note that $\{1,3\}>_{t} Y$ with $\#(Y)=2$ and $2 \notin Y$. Hence, $\{2\}>_{t}\{1,3\}>_{t} Y$ so that the claim holds for any $Y$ with $\#(Y)=2$. Let us finally address the case for which $Y$ satisfies $\#(Y) \geq 3$. It turns out that $\{1,3\}>_{t} Y$ for any such $Y$ as long as $2 \notin Y$ since any candidate in $Y$ different from 1 and 3 is strictly less preferred than 1 and 3. Therefore, $\{2\}>_{t}\{1,3\}>_{t} Y$ which is equivalent to $Y \cup\{2\}>_{t} Y$ as wanted.

d. Candidate $i$. The claim immediately holds for any $Y$ with $\#(Y)=1$. Assume from now on that $\#(Y) \geq 2$. By assumption, $\{i\}>_{t}\{1, \ldots, i-1, i+1\}$. Note that $\{1, \ldots, i-$ $1, i+1\}>_{t} Y$ for any $Y$ with $\#(Y) \geq i$ and such that $i \notin Y$. Hence, $\{i\}>_{t} Y$ which is equivalent to $Y \cup\{i\}>_{t} Y$ as wanted. In order to conclude the proof for candidate $i$, one needs to ensure that $\{i\} \cup Y>_{t} Y$ for any $Y$ with $2 \leq \#(Y) \leq i-1$. However, note that $\{i\}>_{t}\{1, \ldots, i-1, i+1\}$ (by assumption) and $\{1, \ldots, i-1, i+1\}>_{t}\{1, \ldots, i-2, i+1\}$. Indeed, by assumption $\{i-1\}>_{t}\{1, \ldots, i-2, i\}$ and by definition $\{1, \ldots, i-2, i\}>_{t}$ $\{1, \ldots, i-2, i+1\}$ so that $\{i-1\}>_{t}\{1, \ldots, i-2, i+1\} \Longleftrightarrow\{1, \ldots, i-2, i-1, i+1\}>_{t}\{1, \ldots, i-$ $2, i+1\}$. Moreover, $\{1, \ldots, i-2, i+1\}>_{t} Y$ with $\#(Y)=i-1$ as long as $Y \nsubseteq U C S_{t}(i)$. Hence, it follows that

$$
\{i\}>_{t}\{1, \ldots, i-1, i+1\}>_{t}\{1, \ldots, i-2, i+1\}>_{t} Y,
$$

with $\#(Y)=i-1$ as wanted. As similar argument extends the proof for any set of candidates $Y$ with $2 \leq \#(Y) \leq i-2$ concluding the proof.

Building on Proposition 2, we can now easily check whether a utility vector satisfies the sufficient condition for sincerity.

\section{Examples:}

Example 1: Let $k=4$ with $u_{t}=(3, x, 1,0)$. Hence, candidate 2 is strong if and only if $x>\frac{1+3}{2}=2$. As the set of top candidates equals candidates 1 and 2 (all candidates but the two last preferred ones), the previous inequality is enough to ensure sincerity of the voters. 
ExAmple 2: Let $k=5$ with $u_{t}=(4, y, x, 1,0)$ with $4>y>x>1$. In order to ensure that the set of strong candidates equals the set of top candidates, the utility levels $(x, y)$ must belong to the set $\mathcal{U}=\left\{(x, y) \in \mathbb{R}^{2} \mid 3 x-5>y>\frac{4+x}{2}, y>x>1\right\}$

EXAmple 3: Let the $t$-voter's preferences be such that $u_{t}(i)=(k-i)^{\alpha}$. When $k=4$, the sufficient condition for sincerity is satisfied when $\alpha \in(0,1)$ whereas there is no strong candidate when $\alpha \notin(0,1)$. When $k \geq 5$, the sufficient condition is never satisfied since the conditions for candidates 2 and 3 being strong are incompatible. Indeed, assume that $S_{t}(\mathcal{K})=T_{t}(\mathcal{K})$ so that candidates 2 and 3 are strong. By Proposition 2, it must be respectively the case that $(k-2)^{\alpha}>\frac{(k-1)^{\alpha}+(k-3)^{\alpha}}{2}$ and that $(k-3)^{\alpha}>\frac{(k-1)^{\alpha}+(k-2)^{\alpha}+(k-4)^{\alpha}}{3}$. The first inequality just holds when $\alpha \in(0,1)$ whereas the second one does not in this interval proving the claim.

\section{Skipping a Candidate with a Positive Share of Votes: Ordinal Preferences}

Take a LPG with $K=\{1,2,3,4\}$. Voters' preference orderings are depicted in the next table:

\begin{tabular}{|c|c|c|c|c|}
$t_{1}$ & $t_{2}$ & $t_{3}$ & $t_{4}$ & $t_{5}$ \\
\hline 1 & 1 & 2 & 3 & 4 \\
2 & 4 & 1 & 4 & 1 \\
3 & 2 & 4 & 1 & 2 \\
4 & 3 & 3 & 2 & 3 \\
\hline
\end{tabular}

Besides the distribution of types satisfies

$$
r\left(t_{1}\right)=0.125, r\left(t_{2}\right)=0.25, r\left(t_{3}\right)=0.325, r\left(t_{4}\right)=0.125 \text {, and } r\left(t_{5}\right)=0.175 \text {. }
$$

In the strategy function $\sigma_{2}$ depicted as follows,

$$
\sigma_{2}=(\{1,3\},\{1\},\{1,2\},\{3,4\},\{4\}) .
$$

one can see $t_{1}$-voters violate no-skipping sincerity as they approve of 1 and 3 but not 2, their second preferred candidate. Given these strategies, candidate 1 is the unique front-runner of the election.

The main objective of this section is to prove that for some suitable restriction over the intensities of preferences of $t_{1}$-voters,$\sigma_{2}$ constitutes a strict large equi- 
librium in which the $t_{1}$-voters play their unique best response. Importantly, the sufficient condition for approving of a candidate with no votes (introduced in the previous section) holds in the sense that the top candidates are strong.

The Poisson structure makes that 2 is involved only in pivots in which 1 is included and whereas the most likely pivotal events involving 3 include both candidates 1 and 4 . Hence, the best response for $t_{1}$ voters is to approve of her first preferred and third preferred candidate.

We assume that $u_{t_{1}}$ satisfies $u_{t_{1}}(3)>0.279 u_{t_{1}}(1)+0.721 u_{t_{1}}(4)$. Note that this restriction does not imply a restriction on $u_{t_{2}}(2)$.

Proposition 3. $\sigma_{2}$ is a strict large equilibrium which skipping is a strict best response.

Corollary 4. Approving/skipping a candidate with a positive share of votes need not depend on the voters' intensities towards this candidate.

Proof. Within the proof, we write $u(k)$ for $u_{t_{1}}(k)$ in order to simplify the notation, as the rest of the voters' best responses hinging uniquely on their ordinal preferences. In order to determine whether the voters approve each of the candidates, we describe the pivot probabilities in which each of these candidates is included. Within the proof, we use the Magnitude Equivalence Theorem included in appendix B to compute the pivot magnitudes.

\section{Pivots in which 1 is involved.}

Due to the correlations of the scores, the pivot with the highest magnitude in which 1 is included is the one between 1 and 4 . Indeed, the magnitude of pivot $(1,4)$ equals $-\left(\sqrt{r\left(t_{1}\right)+r\left(t_{2}\right)+r\left(t_{3}\right)}-\sqrt{r\left(t_{4}\right)+r\left(t_{5}\right)}\right)^{2}=-0.083$ and the rest of the pivot outcomes have a magnitude lower than the one associated to pivot $(1,3)$ which equals $-\left(\sqrt{r\left(t_{2}\right)+r\left(t_{3}\right)}-\sqrt{r\left(t_{4}\right)}\right)^{2}=-0.168$. Hence the voters who prefer 1 to $4\left(t_{1}, t_{2}\right.$ and $\left.t_{3}\right)$ approve of 1 whereas the ones who prefer 4 to 1 give no point to $1\left(t_{4}\right.$ and $\left.t_{5}\right)$.

\section{Pivots in which 4 is involved.}

The same claim applies to the pivots in which 4 is included. Indeed, the magnitude of pivot $(1,4)$ equals -0.083 whereas the rest of the pivot magnitudes in which 4 is present have a magnitude lower than -0.168 , the magnitude of pivot $(3,4)$. Hence, $t_{4}$ and $t_{5}$ approve of 4 whereas the rest of the voters give him one point.

\section{Pivots in which 2 is involved.}

In the strategy function $\sigma_{2}$, the set of voters who vote for 2 is a subset of the set of voters who vote for 1 . Therefore candidate 2 being involved in a pivot requires 
that no voter of type $t_{1}$ and $t_{2}$ is present in the election (as they vote $\{1,3\}$ and $\{1\}$ ) as otherwise 1 would have a strictly larger score than 2 . Hence, in these pivot events, the score of candidate 3 is lower than the score of candidate 4 as the only votes he gets are the ones from the ballot $\{3,4\}$. Thus, approving of candidate 2 has an impact in the outcome of the election in two cases (the rest of pivot outcomes being infinitely less likely): if either breaks a tie between candidates 1 and 2 or a threeway tie between candidates 1,2 and 4 . However, the magnitude of pivot $(1,2)$ equals $-r\left(t_{1}\right)-r\left(t_{2}\right)=-0.375$ whereas the magnitude of pivot $(1,2,4)$ equals $-r\left(t_{1}\right)-r\left(t_{2}\right)-$ $\left(\sqrt{r\left(t_{3}\right)}-\sqrt{r\left(t_{4}\right)-r\left(t_{5}\right)}\right)^{2}=-0.3755$.

Hence, all voters anticipate that approving of candidate 2 is pivotal against 1 . Therefore, the $t_{3}$-voters are the only voters approving of 2 as the rest of the voters strictly prefer 1 to 2 .

\section{Pivots in which 3 is involved.}

Given the voters' preferences, the $t_{4}$ voters always approve of 3 (their preferred candidate) whereas the voters of type $t_{2}, t_{3}$ and $t_{5}$ do not approve of this candidate since its their worst preferred choice. As far the decision of $t_{1}$ voters, the Magnitude Equivalence Theorem proves that among the pivot outcomes including 3, the ones with the highest magnitude are the one between 3 and 1 and the one between 3 and 4 and the one between 1,3 and 4 share the same magnitude. Hence, the decision problem of $t_{1}$ voters just focuses on the previously mentioned pivot events.

Letting $p_{31}, p_{34}$ and $p_{314}$ the pivot probabilities, the $t_{1}$ voters' decision problem when deciding between $\{1\}$ and $\{1,3\}$ equals:

$$
U[\{1\}]-U[\{1,3\}]=\left(\frac{u(1)-u(3)}{2}\right) p_{13}+\left(\frac{u(4)-u(3)}{2}\right) p_{34}+\left(\frac{u(1)+u(4)-2 u(3)}{6}\right) p_{124} .
$$

The pivot events can be described by

$$
\begin{array}{r}
X_{3,1}=\left\{x \in Z(C) \mid s_{x}^{k}=(a, b, a, a-k) \text { or } s_{x}^{k}=(a, b, a+1, a-k)\right. \\
\text { for each } \left.a, b, k \in \mathbb{N}^{+}, k \in\{0, \ldots, a\}, \text { with } b+1<a\right\}, \\
X_{3,4}=\left\{x \in Z(C) \mid s_{x}^{k}=(a-k, b, a, a+1) \text { or } s_{x}^{k}=(a-k, b, a+1, a+1)\right. \\
\text { for each } \left.a, b, k \in \mathbb{N}^{+}, k \in\{1, \ldots, a\}, \text { with } b+1<a\right\},
\end{array}
$$


and

$$
\begin{array}{r}
X_{1,3,4}=\left\{x \in Z(C) \mid s_{x}=(a, b, a, a+1) \text { or } s_{x}=(a-1, b, a, a)\right. \\
\text { for each } \left.a, b \in \mathbb{N}^{+}, \text {with } b+1<a\right\} .
\end{array}
$$

We let $W_{0}=\left\{x \in Z(C) \mid s_{x}=(a, b, a, a)\right.$ for each $a, b \in \mathbb{N}^{+}$, with $\left.b+1<a\right\}$. Since all the events within each of these sets differ by a single translation from $W_{0}$, the offset theorem implies that

$$
\begin{aligned}
& \lim _{n \rightarrow \infty} \frac{p_{31}}{P\left[W_{0} \mid n \tau\right]}=\frac{1+\alpha(4)}{1-\alpha(4)}, \\
& \lim _{n \rightarrow \infty} \frac{p_{34}}{P\left[W_{0} \mid n \tau\right]}=\frac{1+\alpha(1)}{1-\alpha(1)},
\end{aligned}
$$

and

$$
\lim _{n \rightarrow \infty} \frac{p_{314}}{P\left[W_{0} \mid n \tau\right]}=\alpha(1)^{-1}+2 \alpha(1) .
$$

Since $\alpha(1)=\sqrt{\frac{\tau(3,4)}{\tau(1)+\tau(1,2)}}=0.466$ and $\alpha(4)=\sqrt{\frac{\tau(1,3)}{\tau(4)}}=0.845$, it follows that $(1)$ is equivalent to

$$
0.279 u(1)+0.721 u(4)-u(3)
$$

Hence, the $t_{1}$ voters approve of candidate 3 when the previous expression is strictly negative which is equivalent to $0.279 u(1)+0.721 u(4)<u(3)$ as wanted.

\section{Discussion}

As discussed in the introduction, Laslier [21] proves that no-skipping sincerity un$\operatorname{der} A V$ is ensured in a large election model. More precisely, in such a model, if there is no tie in the expected scores of the candidates, the voter's best response is sincere (Corollary 1 page 16). We now discuss the difference between such a model and the current Poisson structure, in order to underline the driving force behind the lack of sincerity the previous examples.

In contrast with our model, Laslier [21] assumes that there is a finite number of voters. However, voters know that with some positive probability, each point assigned to each candidate might be erased with positive probability. This implies that, given the voters' best responses, the scores of the different candidates are independent random variables. The author can hence prove that, when $n$ is large 
enough, for every three distinct candidates $i, j$ and $l$, the pivot probabilities satisfy the next condition:

$$
\text { if } \rho(i)>\rho(j) \text { then } \lim _{n \rightarrow \infty} \frac{p_{j l}}{p_{i l}}=0 .
$$

It follows that, in an equilibrium with a unique leading candidate $i$, a voter approves of candidate $j \neq i$ if and only if the candidate is preferred to the front-runner (as, conditional on the event of being in a pivot, $j$ is involved almost surely in a pivot against i). Similarly, a voter approves of the front-runner if and only if he prefers the front-runner to the candidate with the second highest score. These best responses follow the leader rule as every voter takes only into account to determine her best response the leading candidate of the election. This naturally triggers the no-skipping sincerity of voters: they all vote for one of the two leading candidates and for the rest of candidates they prefer to.

In Poisson Games, the condition 2 is violated and this violation leads to situations such as the one depicted in Examples 1 and 2. Note that, in Laslier [21]'s model, the strategies depicted by Example 2 (and a similar claim applies to Example 1) do not constitute an equilibrium. Indeed, given $\sigma_{2}$ and $r$, the expected score distribution $\rho=(\rho(j))_{j \in K}$ satisfies:

$$
\rho(1)=0.7>\rho(2)=0.325>\rho(4)=0.3>\rho(2)=0.25 \text {. }
$$

The front-runner is candidate 1 so that in Laslier [21]'s model, the $t_{1}$-voters would anticipate that, if approving of any candidate $j$ distinct from 1 , the most likely pivot outcome in which $j$ is involved is against 1 . Hence, $t_{1}$-voters would not approve of candidate 2 and candidate 3 as they prefer candidate 1 to both 2 and 3 . Therefore, the non-sincerity of the voters is driven by the Poisson structure of the game in the set of equilibria without ties and strictly positive scores ${ }^{15}$.

This does not seem to be a particularly desirable feature of Poisson games as it tends to generate a multiplicity of equilibria under $A V^{16}$. Moreover, it seems to be deeply linked to the correlation between the scores of the candidates that naturally arise in Poisson games under $A V$. Few empirical and experimental works deal with approval voting. These few works seem to confirm the intuition that the voters vote sincerely. Laslier and Van der Straeten [20] report on an experiment on $A V$ and Baujard et al. [4] report on experiments comparing the use of $E V$ under various

\footnotetext{
${ }^{15}$ I would like to thank a referee for pointing this out.

${ }^{16}$ See the discussion on the political significance of a multiplicity of equilibria included in Myerson and Weber [30] and the idea of focal manipulation originally coined by Schelling [38].
} 
scales, including $A V$. Finally, Blais et al. [5, 6] report on internet-based experiments and underline that the voters tend to provide more votes than with plurality voting to small parties and "to favour, big or small, parties that are deemed to be acceptable by many voters".

\section{Sincerity of Scoring Rules}

We now focus on the strategic sincerity of ranked scoring rules (note that $A V$ is a non-ranked scoring rule). Following Myerson [26], a ranked scoring rule in a $k$ candidates election is characterized by some list of numbers $c_{1}, c_{2}, \ldots, c_{k}$ such that $1=c_{1} \geq c_{2} \geq \ldots \geq c_{k}=0$. For instance, Plurality voting is such that $c_{j}=0$ for any

$j \neq 1$. Similarly, the Borda rule satisfies $c_{j}=\frac{k-j}{k-1}$. Negative voting belongs to such a family as $c_{j}=1$ for any $j \neq k$.

As previously discussed, the sincerity of $A V$ in equilibrium of Poisson games has three key aspects:

1. A voter always assigns 1 point to her preferred candidate (Best Candidate Sincerity)

2. A voter always assigns 0 points to her least preferred candidate (Worst Candidate Sincerity)

3. Under some conditions, whenever a voter approves of some candidate, he approves of every candidate he prefers to (No-skipping Sincerity).

We evaluate the sincerity of scoring rules by judging whether they satisfy each of the previous aspects. The fact that no-skipping sincerity need not be satisfied is a simple extension of Myerson (2002)'s examples ${ }^{17}$. However, it is not completely clear whether the other two notions hold: in other words, whether there is some implicit notion of sincerity in rank scoring rules. We now prove that Plurality Voting is the only rule that prevents voters from assigning a strictly positive score to their worst ranked candidate. Similarly, Negative Voting is the unique one that ensures that the voters always assign a score of 1 to their most preferred candidate. These two results give a natural grounding to a well-known result: Approval voting, which ballots are the union of the ones corresponding to these two rules, is the more prone among (ranked and non-ranked) scoring rules to sincerity.

\footnotetext{
${ }^{17}$ An example of this violation is included in Núñez [34] (the working paper version of this work).
} 
The analysis first focuses on worst candidate sincerity and then examines best candidate sincerity.

\section{Worst Candidate Sincerity}

We now prove that there exists an equilibrium under any rank scoring rule in which voters assign a score of $c_{2}$ to their least preferred candidate ${ }^{18}$. Therefore, the unique rank scoring rule that shares the feature of $A V$ according to which a voter never gives a positive score to this candidate is Plurality voting as $c_{2}=0$.

Let $K=\{1,2,3\}$ and $T=\left\{t_{1}, t_{2}\right\}$ with $r\left(t_{1}\right)=p$ so that $r\left(t_{2}\right)=1-p$ for some $0<p<1$.

The $t_{1}$-voters prefer to 1 to 2 and 2 to 3 whereas the $t_{2}$-voters prefer to 1 to 3 and 3 to 2 . They both attach an utility of 1 to their best candidate, $\rho$ to their second candidate and 0 to their least preferred one.

Take the strategy combination $\sigma$ according to which $t_{1} \rightarrow\left\{1,0, c_{2}\right\}$ and $t_{2} \rightarrow$ $\left\{1, c_{2}, 0\right\}$. Given $\sigma$, it follows that the possible outcomes of the election are described by the following set:

$$
X=\left\{x \in Z(C) \mid s_{x}=\left(a+b, b c_{2}, a c_{2}\right) \text { for each } a, b \in \mathbb{N}^{+}\right\} .
$$

As long as $a, b \geq 1$, it follows that $a+b>a c_{2}, b c_{2}$ as $a \geq a c_{2}$ and $b \geq b c_{2}$. In all these events, candidate 1 is the only winner. It follows that both types of voters prefer to assign the maximal score to candidate 1 as each of these ballots ensures the victory of their preferred candidate.

If $a=0$ and $b=0$, every voter abstains. Hence, both types of voters are indifferent between all the ballots that assign 1 point to their preferred candidate as they both ensure the victory of 1 .

If $a=0$ and $b=1$, then $s_{x}=\left(1, c_{2}, 0\right)$. If a voter votes $\left(1,0, c_{2}\right)$, the total score equals $\left(2, c_{2}, c_{2}\right)$ so that 1 is the sole winner of the election. On the contrary, if he votes $\left(1, c_{2}, 0\right)$ the total score equals $\left(2,2 c_{2}, 0\right)$. Given that $c_{2}<1$, all voters are indifferent between both ballots and prefer them to the rest of the ballots.

If $a=1$ and $b=0$, then $s_{x}=\left(1,0, c_{2}\right)$. If a voter votes $\left(1, c_{2}, 0\right)$, the total score equals $\left(2, c_{2}, c_{2}\right)$ so that 1 is the sole winner of the election. On the contrary, if he votes $\left(1,0, c_{2}\right)$ the total score equals $\left(2,0,2 c_{2}\right)$. As $c_{2}<1$, all voters are indifferent between both ballots and prefer them to the rest of available ballots.

\footnotetext{
${ }^{18}$ Note that Myerson [29] proves that there exists a large equilibrium under negative voting in which voters assign one point with positive probability to their least preferred candidate (p.238239).
} 
Hence, the strategy combination $\sigma$ is a large equilibrium of this game.

Lemma 1. If $c_{2}>0$, a voter might assign a strictly positive score to her worst preferred candidate in equilibrium.

Corollary 5. Plurality Voting is the unique rank scoring rule that ensures that the voters assign a score of zero to their worst preferred candidate in equilibrium.

\section{Best Candidate Sincerity}

The definition of strong insincerity for $A V$ entails that "a voter may skip some candidates and does not vote for her preferred choice". The idea of strong insincerity is strongly related to the idea of voting for your most-preferred candidate, that we have denoted best candidate sincerity for rank scoring rules.

There are two possible adaptations of this principle when voters under a scoring rule: a weak version and a strong one.

The weak version states that a rule is insincere if there exists an equilibrium in which some voter gives 0 points to her preferred candidate.

The strong version of this statement is that a rule is insincere on the best candidate, if there exists an equilibrium in which some voter does not give 1 point to her preferred candidate.

We now prove how the different scoring rules satisfy both principles: the weak requirement is satisfied by all rules but Plurality voting whereas Negative voting is the unique one verifying the strong version of this principle.

\section{Weak Best Candidate Sincerity}

In order to understand which rank scoring rules satisfy the weak version, we use the characterization of weakly undominated strategies in scoring rules voting games provided by Dellis [12]. It is not too difficult to prove using her condition that a voter always assign a (weakly) higher score to her most preferred candidate than to her worst preferred one (Lemma 2). Hence, in three candidate elections, Plurality voting is the unique scoring rule under which strong insincerity might arise. More formally,

Lemma 2. If $c_{k-1}>0$, it is weakly dominated for a voter to assign a score of zero to her most preferred candidate. 
Proof. Take a voter with type $t$ with $u_{t}(1)>u_{t}(2)>\ldots>u_{t}(k)$. Take a scoring rule with $c_{k-1}>0$. According to Dellis [12], if there are at least four voters ${ }^{19}$, a ballot $v$ is weakly undominated for a $t$-voter if and only if there is no ballot $w \in C$ such that for every pair of candidates $i$ and $j, u_{t}(i) \geq u_{t}(j)$ implies that $w_{i}-v_{i} \geq w_{j}-v_{j}$.

Let $v$ be any ballot that assigns a score of 0 to candidate 1 (the voter's preferred candidate) so that $v_{1}=0$. By definition of rank scoring rules, it must be the case that $v_{k}=c_{l}$ for some $c_{l}$. As by assumption, $c_{k-1}>0$ it follows that any $c_{l}>0$ as $c_{i} \geq c_{i+1} \forall i$ by definition. Similarly, let $w$ be the ballot that permutes the scores of candidates 1 and $k$ (i.e. $w_{1}=v_{k}=c_{l}$ and $w_{k}=v_{1}=0$ ), the rest of the scores being as in $v$ (i.e. $w_{i}=v_{i}$ for any $i \neq 1, k$ ). It follows that $w_{1}-v_{1}=c_{l}>0$ and $w_{k}-v_{k}=-c_{l}<0$. Moreover, $w_{i}-v_{i}=0$ for any $i \neq 1, k$. Therefore, as $u_{t}(1)>u_{t}(2)>\ldots>u_{t}(k)$, we have that whenever $u_{t}(i) \geq u_{t}(j), w_{i}-v_{i} \geq w_{j}-v_{j}$ so that $w$ weakly dominates $v$, proving the claim.

However, Lemma 2 does not directly imply that in equilibrium of a Poisson game, no voter assigns a score of zero to her most preferred candidates when $c_{k-1}>$ 0. Indeed, De Sinopoli and Pimienta [11] prove that (perfect) equilibria in Poisson games may involve the use of weakly dominated strategies. Nonetheless, as we focus on a voting situation, the probability of every voter abstaining is positive. When every voter abstains, the voter is not anymore indifferent and strictly prefers to assign her preferred candidate a positive score - in equilibrium of a rank scoring rule, no voter assigns a score of zero to her most preferred candidate when $c_{k-1}>0$. We can hence state the following two corollaries.

Corollary 6. If $c_{k-1}>0$, no voter skips her preferred candidate in equilibrium.

Corollary 7. Let $k=3$. Plurality Voting is the unique rule in which a voter might skip her preferred candidate in equilibrium.

\section{Strong Best Candidate Sincerity}

A more stringent requirement seems to be whether a voter assigns a score of 1 to her most preferred candidate. The next example proves that Negative voting is the

\footnotetext{
${ }^{19}$ The characterization of weak undominance with at most three voters differs. Nonetheless, our claim remains valid as assigning a higher score to one's own favorite candidate than to one's own least favorite candidate still weakly dominates assigning a higher score to one's own least favorite candidate than to one's own favorite candidate.
} 
unique rank scoring rule that ensures that this happens in equilibrium (with three candidates).

Let $K=\{1,2,3\}$ and $T=\left\{t_{1}, t_{2}\right\}$ with $u_{t_{1}}=(10,8,0), u_{t_{2}}=(1,0,10)$ and $u_{t_{3}}=$ $(0,10,1)$.

We consider any scoring rule with score vector $\left(1, c_{2}, 0\right)$. Within this section, we let $s=c_{2}$ to simplify the notations. The current example can be extended to a many candidates setting even though the presentation is done for three candidates for simplicity.

If $s \leq 1 / 2$, we assume that

$$
r\left(t_{1}\right)=\frac{3-s}{6}, r\left(t_{2}\right)=\frac{2+s}{6} \text { and } r\left(t_{3}\right)=\frac{1}{6},
$$

whereas if $s>1 / 2$ we assume that

$$
r\left(t_{1}\right)=\frac{1+s^{2}}{6 s}, r\left(t_{2}\right)=\frac{(3-s) s}{6 s} \text { and } r\left(t_{3}\right)=\frac{3 s-1}{6 s} .
$$

Let $\beta$ denote the strategy combination in which the $t_{1}$-voters play the mixed strategy

$$
\beta\left((1, s, 0) \mid t_{1}\right)=q_{s}, \beta\left((s, 1,0) \mid t_{1}\right)=1-q_{s},
$$

whereas the rest of the voters play in pure strategies

$$
\beta\left((s, 0,1) \mid t_{2}\right)=1 \text { and } \beta\left((0,1, s) \mid t_{3}\right)=1 .
$$

Note that in $\beta$, the $t_{1}$ voters cast with some positive probability the ballot $(s, 1,0)$ so that they violate the strong best candidate sincerity. We set $q_{s}=\frac{2-3 s}{3-4 s+s^{2}}$ if $s \leq 1 / 2$ and $q_{s}=\frac{s}{1+s^{2}}$ when $s>1 / 2$ so that, given the voters' strategies, the expected scores of the three candidates are all equal to $\frac{1+s}{3}$.

We now prove that $\beta$ is an equilibrium for any rank scoring rule, proving that the unique rank scoring rule that ensures strong best candidate sincerity is negative voting.

Lemma 3. If $c_{2}<1$, a voter needs not assign a score of one to her most preferred candidate in equilibrium.

Proof. If all the pivot probabilities were equally likely, all voters will cast a ballot that mimics their true preference profile ${ }^{20}$ contradicting the fact that $\beta$ is an equi-

\footnotetext{
${ }^{20}$ See Núñez and Laslier [35] for a general proof of this claim in the closely related Myerson-Weber setting.
} 
librium. As all events occur here in the region where all the offset ratios are close to 1 (in the sense that the most likely event is the tie among the three candidates) we can use the Normal approximation (see Theorem 3 of by Myerson [27] and Myerson [29] for an application). In this Normal approximation for the game of expected size $n$, the components of the realized vote profile $\left(x_{n}(c)\right)_{c \in C}$ are approximated as the integer roundings of independent Normal random variables, where each $x_{n}(c)$ has a mean and a variance both equal to $n \tau_{n}(c)$.

Again, as all offset ratios are close to 1, the probability of any pivot event is roughly equivalent to the probability of candidates $i$ and $j$ being tied for first place (their ratio tends to 1 as $n \rightarrow \infty$ due to the offset theorem). For any large $n$, we set $p_{i j}$ the probability of $i$ and $j$ being for first place.

The indifference of $t_{1}$-voters between ballots $(1, s, 0)$ and $(s, 1,0)$ is equal to

$$
(10-8)(1-s) p_{12}+10 p_{13}+x s p_{23}=(10-8)(s-1) p_{12}+10 s p_{13}+8 p_{23},
$$

which can be rewritten as

$$
4(1-s) p_{12}+10(1-s) p_{13}=8(1-s) p_{23}
$$

which is in turn equivalent to write

$$
4 p_{12}+10 p_{13}=8 p_{23}
$$

Note that the sincerity of $t_{2}$ and $t_{3}$ voters is triggered by the fact that the pivot probabilities are not too different. Take first the case of Plurality voting $(s=0)$. It follows that $r\left(t_{1}\right)=3 / 6, r\left(t_{2}\right)=2 / 6$ and $r\left(t_{3}\right)=1 / 6$ and $q_{0}=2 / 3$. In order to justify the previous equality, one may focus on equilibria of the type $\beta_{n}$ with

$$
\beta_{n}\left((1,0,0) \mid t_{1}\right)=2 / 3+\varepsilon_{n}, \beta_{n}\left((0,1,0) \mid t_{1}\right)=1 / 3-\varepsilon_{n},
$$

whereas the rest of the voters play in pure strategies

$$
\beta_{n}\left((0,0,1) \mid t_{2}\right)=1 \text { and } \beta_{n}\left((0,1,0) \mid t_{3}\right)=1 \text {. }
$$

These strategies lead to the expected vote distribution:

$$
\tau_{n}(1,0,0)=1 / 3+\varepsilon_{n} / 2, \tau_{n}(0,1,0)=1 / 3-\varepsilon_{n} / 2, \text { and } \tau_{n}(0,0,1)=1 / 3
$$


Using the Normal approximation, the number of ballots cast $x(c)$ for each ballot $c$ can be approximated by integer roundings of independent Normal random variables with mean and variance equal to $n \tau_{n}(c)$. Hence, we define the random variables $\Delta_{i, j}^{n}$ that represent the normalized difference of scores between candidates $i$ and $j$ so that $\Delta_{1,2}^{n}=\frac{x_{n}(1,0,0)-x_{n}(0,1,0)}{\sqrt{n}}, \Delta_{1,3}^{n}=\frac{x_{n}(1,0,0)-x_{n}(0,0,1)}{\sqrt{n}}$ and $\Delta_{2,3}^{n}=\frac{x_{n}(0,1,0)-x_{n}(0,0,1)}{\sqrt{n}}$. This leads to $E\left(\Delta_{1,2}^{n}\right)=\varepsilon_{n} \sqrt{n}, E\left(\Delta_{1,3}^{n}\right)=1 / 2 \varepsilon_{n} \sqrt{n}$ and $E\left(\Delta_{2,3}^{n}\right)=-1 / 2 \varepsilon_{n} \sqrt{n}$. Moreover their variances are all close respectively to $2 / 3$. The correlation between $\Delta_{1,2}^{n}$ and $\Delta_{1,3}^{n}$ equals $1 / 2$ whereas the one between $\Delta_{1,2}^{n}$ and $\Delta_{2,3}^{n}$ equals $-1 / 2$.

Remark first that $p_{i j}=P\left[\Delta_{i, j}^{n}=0 \cap \Delta_{i, k}^{n}>0 \mid n \tau\right]$ for any $i, j, k \in \mathcal{K}$.

In order to ensure that (3) holds, we compute the probabilities of the different pivot outcomes. The pivot between 1 and 2 occurs when $\Delta_{1,2}^{n} \approx 0$ and $\Delta_{1,3}^{n}$ is positive. However, when $\Delta_{1,2}^{n}$ is close to zero, their joint distribution is approximatively normal with mean 0 and variance $1 / 2$. Letting the $F(x, \mu, \sigma)$ and $f(x, \mu, \sigma)$ respectively denote the cumulative and density at $x$ for a Normal distribution with mean $\mu$ and variance $\sigma$, we have that

$$
p_{12} \approx(1 / \sqrt{n}) f\left(0, \varepsilon_{n} \sqrt{n}, 2 / 3\right)(1-F(0,0,1 / 2)) .
$$

Similarly, the pivot outcome between 1 and 3 occurs when $\Delta_{1,3}^{n} \approx 0$ and $\Delta_{1,2}^{n}$ is positive. But when $\Delta_{1,3}^{n}$ is close to zero, their joint distribution follows a Normal distribution of mean $3 / 4 \varepsilon_{n} \sqrt{n}$ and variance $1 / 2$, so that:

$$
p_{13} \approx(1 / \sqrt{n}) f\left(0,1 / 2 \varepsilon_{n} \sqrt{n}, 2 / 3\right)\left(1-F\left(0,3 / 4 \varepsilon_{n} \sqrt{n}, 1 / 2\right)\right) .
$$

Similarly, the pivot outcome between 2 and 3 occurs when $\Delta_{2,3}^{n} \approx 0$ and $\Delta_{1,2}^{n}$ is negative. But when $\Delta_{2,3}^{n}$ is close to zero, their joint distribution follows a Normal distribution of mean $3 / 4 \varepsilon_{n} \sqrt{n}$ and variance $1 / 2$, so that:

$$
p_{23} \approx(1 / \sqrt{n}) f\left(0,-1 / 2 \varepsilon_{n} \sqrt{n}, 2 / 3\right) F\left(0,3 / 4 \varepsilon_{n} \sqrt{n}, 1 / 2\right) .
$$

The indifference condition (3) is equivalent to $\varepsilon_{n}=-0.2746 / \sqrt{n}$ which leads to the following pivot probabilities

\begin{tabular}{|c|c|c|}
\hline$p_{12}$ & $p_{13}$ & $p_{23}$ \\
\hline $0.2748 / \sqrt{n}$ & $0.1993 / \sqrt{n}$ & $0.3865 / \sqrt{n}$ \\
\hline
\end{tabular}

Note that these probabilities ensure that the $t_{2}$-voters and the $t_{3}$ voters vote as in $\beta$. 
Similar estimation techniques (fully described in the appendix) prove that there exists some $\varepsilon_{n}$ for each value of $s$ for which the indifference condition holds. Letting $\varepsilon_{n}^{s}=\rho_{s} / \sqrt{n}$ for each rule with vector $(1, s, 0)$ we have the following tables for some the scoring rules that describe the sequence of equilibria that justify the voters' decisions in $\beta_{n}$ concluding the proof.

\begin{tabular}{|c|c|c|c|c|c|c|}
\hline $\mathrm{s}$ & 0 & 0.1 & 0.2 & 0.3 & 0.4 & 0.5 \\
\hline$\rho_{s}$ & -0.2746 & -0.3135 & -0.3629 & -0.4266 & -0.513 & -0.6384 \\
\hline $\mathrm{s}$ & 0.6 & 0.7 & 0.8 & 0.9 & 0.95 & 0.99 \\
\hline$\rho_{s}$ & -0.959 & -1.432 & -2.2478 & -4.414 & -8.598 & -41.577 \\
\hline
\end{tabular}

Corollary 8. Negative Voting is the unique rank scoring rule that ensures that the voters assign a score of one to their most preferred candidate in equilibrium.

\section{Conclusion}

This work discusses the incentives for sincerity under $A V$ in Poisson voting games. It proves that none of the existing definitions in the literature are satisfied in this game-theoretical framework. Broadly speaking, these definitions are based on the idea of no-skipping: a voter approves of any candidate preferred to her worstpreferred approved candidate.

We identify the two main situations in which a rational voter skips a candidate under $A V$. First, if no voter votes for a given candidate, it is rational not to vote for him as the pivot events for some set of cardinal utilities. Second, if a candidate has a positive (expected) vote share, it might be the case that voting for him might be pivotal against one's top preferred alternative while approving of a least preferred candidate might be pivotal against some other candidate. While we are able to find a sufficient condition to prevent the first type of insincerity, the second type of insincerity seems deeply related to the correlations between the scores of the candidates present in Poisson games. Indeed, we prove that a voter never skips a strong candidate ${ }^{21}$ if this candidate has no votes. Hence, requiring that all candidates but the two last ranked ones are strong is enough to prevent this sort of insincerity. In a sense, this condition generalizes the well-known result under $A V$

\footnotetext{
${ }^{21} \mathrm{~A}$ candidate $i$ is strong for a voter if the voter prefers the victory of this candidate over the victory of any other set of candidates (expect the sets that uniquely include candidates strictly preferred to i).
} 
that a voter always approves of her most preferred candidate as a candidate ranked first is always strong. Moreover, it proves that the voters do not skip/approve of a candidate on the sole basis of their probability of winning (as it is the case under the most common used rule, Plurality rule) but also take into account their intensity of preferences towards it.

Furthermore, we set up a comparison with the strategic sincerity under any of the ranked scoring rules. We prove that with three alternatives the unique rule that ensures that the voters do not assign a positive score point to her last preferred candidate is Plurality Voting; similarly, only the rule of Negative (or Anti-Plurality) can prevent voters from not assigning the maximal number of points to their most preferred alternative. As Approval Voting can be thought as the union of both Plurality and Negative voting (in the sense of the ballots under $A V$ ), we give a clear rationale for the incentives for sincerity given by the approval rule.

\section{References}

[1] D. Ahn and S. Oliveros. Approval Voting and Scoring Rules with Common Values. mimeo, University of California, Berkeley, 2011.

[2] M.A. Ballester and P. Rey-Biel. Does Uncertainty Lead to Sincerity? Simple and Complex Voting Mechanisms. Social Choice and Welfare, 33:477-494, 2009.

[3] S. Barbera. Strategy-proof Social Choice. mimeo, Barcelona Economics Working Paper Series, 420., 2010.

[4] A. Baujard, F. Gavrel, H. Igersheim, J.-F. Laslier, and I. Lebon. Who's Favored by Evaluative Voting? An Experiment During the 2012 French Presidential Elections. mimeo, 2013.

[5] A. Blais, M. Héroux-Legault, L. Stephenson, W. Cross, and E. Gidengil. Assessing the psychological and mechanical impact of electoral rules: A quasiexperiment. Electoral Studies, 31:829-37, 2012.

[6] A. Blais, J.-F. Laslier, and K. Van Der Straeten. Vote au pluriel: How people vote when offered to vote under different rules? PS: Political Science and Politics,, forthcoming, 2013.

[7] L. Bouton and M. Castanheira. One Person, Many Votes: Divided Majority and Information Aggregation. Econometrica, 80:43-87, 2012. 
[8] S. Brams. Mathematics and Democracy: Designing Better Voting and Fair-Division Procedures. Princeton, NJ: Princeton University Press., 2008.

[9] S. Brams and P.C. Fishburn. Approval Voting. Birkhauser, Boston, 1983-2007.

[10] F. De Sinopoli, B. Dutta, and J-F. Laslier. Approval Voting: Three Examples. International Journal of Game Theory, 38:27-38, 2006.

[11] F. De Sinopoli and C. Gonzalez Pimienta. Undominated (and) perfect equilibria in poisson games. Games and Economic Behaviour, 66:775-784, 2009.

[12] A. Dellis. Weak Undominance in Scoring Rule Elections. Mathematical Social Sciences, 59:110-119, 2010.

[13] K. Dowding and M. van Hees. In Praise of Manipulation. British Journal of Political Science, 38:1-15, 2008.

[14] U. Endriss. Sincerity and Manipulation under Approval Voting. Theory and Decision, 74:335-355, 2013.

[15] T. Feddersen and W. Pesendorfer. The Swing Voter's Curse. American Economic Review, 86:408-424, 1996.

[16] T. Feddersen and W. Pesendorfer. Voting Behavior and Information Aggregation in Elections with Private Information. Econometrica, 65:1029-1058, 1997.

[17] T. Feddersen and W. Pesendorfer. Convicting the Innocent: The Inferiority of Unanimous Jury Verdicts under Strategic Voting. American Political Science Review, 92:22-35, 1998.

[18] J. Goertz and F. Maniquet. On the Informational Efficiency of Approval Voting. Journal of Economic Theory, 146:1464-1480, 2011.

[19] V. Krishna and J. Morgan. Overcoming Ideological Bias in Elections. Journal of Political Economy, forthcoming, 2012.

[20] J-F. Laslier and K. Van der Straeten. A live experiment on approval voting. Experimental Economics, 11:97-105, 2008.

[21] J.F. Laslier. The Leader Rule: Strategic Approval Voting in a Large Electorate. Journal of Theoretical Politics, 21:113-136, 2009. 
[22] J.F. Laslier and R. Sanver. The Basic Approval Voting Game. In J.F. Laslier and R. Sanver, editors, Handbook on Approval Voting. Heildelberg: Springer-Verlag, 2010.

[23] A. McLennan. Manipulation in Elections with Uncertain Preferences. Journal of Mathematical Economics, 47:370-375, 2011.

[24] S. Merill III and J. Nagel. The Effect of Approval Balloting on Strategic Voting Under Alternative Decision Rules. American Political Science Review, 81:509$524,1987$.

[25] R. Myerson. Extended Poisson Games and the Condorcet Jury Theorem. Games and Economic Behavior, 25:111-131, 1998.

[26] R. Myerson. Theoretical Comparisons of Electoral Systems. European Economic Review, 43:671-697, 1999.

[27] R. Myerson. Large Poisson Games. Journal of Economic Theory, 94:7-45, 2000.

[28] R. Myerson. Comparison of Scoring Rules in Poisson Voting Games. Journal of Economic Theory, 103:219-251, 2002.

[29] R. Myerson. Comparison of Scoring Rules in Poisson Voting Games. Journal of Economic Theory, 103:219-251, 2002.

[30] R. Myerson and R.J. Weber. A Theory of Voting Equilibria. American Political Science Review, 87:102-114, 1993.

[31] R.G. Niemi. The Problem of Strategic Behavior under Approval Voting. American Political Science Review, 78:952-958, 1984.

[32] M. Núñez. Approval Voting on Large Election Models. In J.F. Laslier and R. Sanver, editors, Handbook on Approval Voting. Heildelberg: Springer-Verlag, 2010.

[33] M. Núñez. Condorcet Consistency of Approval Voting: A Counter Example on Large Poisson Games. Journal of Theoretical Politics, 22:64-84, 2010.

[34] M. Núñez. Sincere Scoring Rules. THEMA 2010-02 Working papers, 2010.

[35] M. Núñez and J.-F. Laslier. Preference Intensity representation: Strategic Overstating in Large Elections. Social Choice and Welfare, forthcoming, 2013. 
[36] P. Pathak and T. Sonmez. School Admission's Reform in Chicago and England: Comparing Mechanisms by their Vulnerability to Manipulation. American Economic Review, forthcoming, 2012.

[37] R. Sanver. Approval as an Intrinsic Part of Preference. In J.F. Laslier and R. Sanver, editors, Handbook on Approval Voting. Heildelberg: Springer-Verlag, 2010.

[38] T.C. Schelling. The Strategy of Conflict. Harvard, Cambrige University Press, 1960.

[39] R. Weber. Approval Voting. Journal of Economic Perspectives, 9:39-49, 1995.

[40] A. Wolitzky. Fully Sincere Voting. Games and Economic Behaviour, 67:720-735, 2009.

\section{A Proof of Lemma 2}

We now describe the estimation techniques for the pivotal probabilities in Lemma 2. Indeed, as all events occur in the equilibrium $\beta$ in the region where all the offset ratios are close to 1 (in the sense that the most likely event is the tie among the three candidates) we can use the Normal approximation (see Theorem 3 of by Myerson [27] and Myerson [29] for an application). In this Normal approximation for the game of expected size $n$, the components of the realized vote profile $\left(x_{n}(c)\right)_{c \in C}$ are approximated as the integer roundings of independent Normal random variables, where each $x_{n}(c)$ has a mean and a variance both equal to $n \tau_{n}(c)$.

For each rank scoring rule with vector $(1, s, 0)$, we consider sequences of equilibria $\beta_{n}$ converging towards $\beta$ in the following sense:

$$
\beta_{n}\left((1, s, 0) \mid t_{1}\right)=q_{s}+\varepsilon_{n}, \beta_{n}\left((s, 1,0) \mid t_{1}\right)=1-q_{s}-\varepsilon_{n},
$$

whereas the rest of the voters play in pure strategies

$$
\beta_{n}\left((s, 0,1) \mid t_{2}\right)=1 \text { and } \beta_{n}\left((0,1, s) \mid t_{3}\right)=1 \text {. }
$$

We define the random variables $\Delta_{i, j}^{n}$ that represent the normalized difference of 
scores between candidates $i$ and $j$ :

$$
\Delta_{i j}^{n}=\frac{s(i)-s(j)}{\sqrt{n}} .
$$

It follows that the probability of a pivot outcome $p_{i j}$ is roughly equivalent to:

$$
p_{i j} \approx(1 / \sqrt{n}) P\left[\Delta_{i j}^{n}=0\right] P\left[\Delta_{i k}^{n}>0 \mid \Delta_{i j}^{n}=0\right] .
$$

We hence need to determine the distribution of each $\Delta_{i j}^{n}$ and the conditional distribution of $\Delta_{i k}^{n}$ given that $\Delta_{i j}^{n}=0$ for any triple $i, j, k$.

The rest of this section presents these distributions and is hence divided in two sections $(s \leq 1 / 2$ and $s>1 / 2)$ since the elections in consideration are different for these two cases.

\section{Rank scoring rules with $s \leq 1 / 2$}

Each of the random variables $\Delta_{i j}^{n}$ follows a Normal random variable $N\left(\mu, \sigma^{2}\right)$ as follows:

$\Delta_{12}^{n} \sim \mathcal{N}\left(\left(\frac{3-s}{3}\right)(1-s) \varepsilon_{n} \sqrt{n}, \frac{1}{6}\left(4-7 s+7 s^{2}\right)\right)$,

$\Delta_{13}^{n} \sim \mathcal{N}\left(\left(\frac{3-s}{6}\right)(1-s) \varepsilon_{n} \sqrt{n}, \frac{1}{6}(s-2)^{2}\right)$,

$\Delta_{23}^{n} \sim \mathcal{N}\left(-\left(\frac{3-s}{6}\right)(1-s) \varepsilon_{n} \sqrt{n}, \frac{1}{6}\left(4-s+4 s^{2}\right)\right)$.

The correlation between $\Delta_{12}^{n}$ and $\Delta_{13}^{n}$ equals $\frac{2-5 s+2 s^{2}}{(s-2) \sqrt{4-7 s+7 s^{2}}}$ and the one between $\Delta_{12}^{n}$ and $\Delta_{23}^{n}$ is equal to $\frac{-2+2 s-5 s^{2}}{\sqrt{4-s+4 s^{2}} \sqrt{4-7 s+7 s^{2}}}$.

Given the distributions of the variables $\Delta_{i j}^{n}$ and its correlations, the different conditional distributions follow Normal random variables of the following form

$\Delta_{12}^{n} \mid \Delta_{13}^{n}=0 \sim \mathcal{N}\left(\left(\frac{3-4 s+s^{2}}{4-2 s}\right) \varepsilon_{n} \sqrt{n}, \frac{1}{2}\left(1-s+s^{2}\right)\right)$,

$\Delta_{13}^{n} \mid \Delta_{12}^{n}=0 \sim \mathcal{N}\left(\left(\frac{\left(3-s-3 s^{2}+s^{3}\right) s}{2\left(4-7 s+7 s^{2}\right)}\right) \varepsilon_{n} \sqrt{n}, \frac{(s-2)^{2}\left(1-s+s^{2}\right)}{2\left(4-7 s+7 s^{2}\right)}\right)$,

$\Delta_{12}^{n} \mid \Delta_{23}^{n}=0 \sim \mathcal{N}\left(\left(\frac{6-8 s+5 s^{2}-4 s^{3}+s^{4}}{8-2 s+8 s^{2}}\right) \varepsilon_{n} \sqrt{n}, \frac{(s-2)^{2}\left(1-s+s^{2}\right)}{\left.8-2 s+8 s^{2}\right)}\right)$.

\section{Rank scoring rules with $s \geq 1 / 2$}

Each of the random variables $\Delta_{i j}^{n}$ follows a Normal random variable $N\left(\mu, \sigma^{2}\right)$ as follows:

$$
\begin{aligned}
& \Delta_{12}^{n} \sim \mathcal{N}\left(\left(\frac{1+s^{2}}{3 s}\right)(1-s) \varepsilon_{n} \sqrt{n}, \frac{1}{6}\left(1+s^{2}\right)\right), \\
& \Delta_{13}^{n} \sim \mathcal{N}\left(\left(\frac{1+s^{2}}{6 s}\right)(1-s) \varepsilon_{n} \sqrt{n}, \frac{1}{6}\left(4-7 s+7 s^{2}\right)\right),
\end{aligned}
$$


$\Delta_{23}^{n} \sim \mathcal{N}\left(-\left(\frac{1+s^{2}}{6 s}\right)(1-s) \varepsilon_{n} \sqrt{n}, \frac{1}{6}\left(7-7 s+4 s^{2}\right)\right)$.

The correlation between $\Delta_{12}^{n}$ and $\Delta_{13}^{n}$ equals $\frac{-1+s+2 s^{2}}{(1+s) \sqrt{4-7 s+7 s^{2}}}$ and the one between $\Delta_{12}^{n}$ and $\Delta_{23}^{n}$ is equal to $\frac{-2+s}{\sqrt{7-7 s+4 s^{2}}}$.

Given the distributions of the variables $\Delta_{i j}^{n}$ and its correlations, the different conditional distributions follow Normal random variables of the following form $\Delta_{12}^{n} \mid \Delta_{13}^{n}=0 \sim \mathcal{N}\left(\left(\frac{(s-1)\left(1+s^{2}\right)\left(3-5 s+4 s^{2}\right)}{2 s\left(4-7 s+7 s^{2}\right)}\right) \varepsilon_{n} \sqrt{n}, \frac{1+s+s^{3}+s^{4}}{8-14 s+14 s^{2}}\right)$, $\Delta_{13}^{n} \mid \Delta_{12}^{n}=0 \sim \mathcal{N}\left(\frac{(s-1)^{2}\left(1+s^{2}\right)}{2 s(1+s)} \varepsilon_{n} \sqrt{n}, \frac{1-s+s^{2}}{2}\right)$, $\Delta_{12}^{n} \mid \Delta_{23}^{n}=0 \sim \mathcal{N}\left(\left(\frac{(s-1)\left(1+s^{2}\right)\left(2+\frac{(s-2)(s+1)}{\left.7-7 s+4 s^{2}\right)}\right)}{6 s} 8-2 s+8 s^{2}\right) \varepsilon_{n} \sqrt{n}, \frac{1+s+s^{3}+s^{4}}{\left.14-14 s+8 s^{2}\right)}\right)$.

\section{B Computing Magnitudes of Pivot Outcomes in Large Poisson Games}

This note summarizes the main results in the computation of magnitudes in this type of games. Notice that the magnitude of an outcome must be inferior or equal to zero, since the logarithm of a probability is never positive.

If one can show that a pivot between one pair of candidates has a magnitude that is strictly greater than the magnitude of a pivot between another pair of candidates, then the latter becomes infinitely less likely as the expected number of voters goes to infinity. That is to say, given two subsets $Y$ and $Y^{\prime}$ of the set of candidates $K$, for any pair of outcomes pivot $(Y)$ and $\operatorname{pivot}\left(Y^{\prime}\right) \in Z(C)$, if

$$
\mu[\operatorname{pivot}(Y)]>\mu\left[\operatorname{pivot}\left(Y^{\prime}\right)\right]
$$

then we know that the pivot outcome between candidates in $Y$ is infinitely more likely than the pivot outcome between candidates in $Y^{\prime}$, i.e.

$$
\lim _{n \rightarrow \infty} \frac{P\left[\operatorname{pivot}(Y)^{\prime}\right]}{P[\operatorname{pivot}(Y)]}=0
$$

The magnitude theorem (Myerson [27]) sets up a method to compute such a limit as the solution of a maximization problem with a concave and smooth objective function. The dual magnitude theorem or DMT (Myerson [28]) gives a method to compute magnitudes of outcomes that can be defined by linear inequalities involving the vote profile $x=(x(c))_{c \in C}$. Finally, the magnitude equivalence theorem or MET 
(Núñez [33]) states a simple manner of computing the magnitude of pivot outcomes.

Both the MET and the DMT are now presented as they are used to compute the magnitudes in the examples presented within the paper. We first give the definition of offset ratio of an outcome that will be necessary throughout.

For any outcome $B \subset Z(C)$ and any ballot $c \in C$, the ratio $B(c) / n \tau_{n}(c)$ is called the $c$-offset ratio of $B$ when $n \tau_{n}$ is the vote distribution. That is, the $c$-offset is a ratio which describes the number of players who vote for ballot $c$ as a fraction of the expected number of voters who were supposed to cast ballot $c$.

For any ballot $c \in C$, we say that $\alpha(c)$ is the limit of $c$-offsets ${ }^{22}$ in the sequence of outcomes $\left\{B_{n}\right\}_{n \rightarrow \infty}$ iff $\left\{B_{n}\right\}_{n \rightarrow \infty}$ has a finite magnitude and, for every major sequence of points $\left\{b_{n}\right\}$ in $\left\{B_{n}\right\}_{n \rightarrow \infty}$, we have

$$
\alpha(c)=\lim _{n \rightarrow \infty} \frac{B_{n}(c)}{n \tau_{n}(c)}=\frac{B(c)}{n \tau(c)} \text { with } \tau(c)=\lim _{n \rightarrow \infty} \tau_{n}(c) \text { and } B(c)=\lim _{n \rightarrow \infty} \tau_{n}(c) \text {. }
$$

Theorem 2. [Dual Magnitude Theorem, Myerson [28]]Given the vote profile x, let $B \subset$ $Z(C)$ be an outcome defined by

$$
B=\left\{\sum_{c \in C} a_{k}(c) x(c) \geq 0 \forall k \in J\right\},
$$

in which $J$ is a finite set and parameters $a_{k}(c)$ are given for every $k \in J$ and $c \in C$. Suppose that $\lambda \in \mathbb{R}^{C}$ is an optimal solution to the problem

$$
\min _{\lambda} \sum_{c \in C} \tau(c)\left(\exp \left(\sum_{k} \lambda_{k} a_{k}(c)\right)-1\right) \quad \text { s.t. } \lambda_{k} \geq 0, \forall k \in J .
$$

Then the optimal value of the objective function $(F)$ coincides with the magnitude $\mu[B]$ of the outcome $B \in Z(C)$ and the limits of the c-offset ratios associated are such that

$$
\alpha(c)=\exp \left(\sum_{k} \lambda_{k} a_{k}(c)\right) \text {, for all } c \in C .
$$

\footnotetext{
${ }^{22}$ Technically speaking, $\alpha(c)$ is the limit of the major $c$-offsets. A sequence $\left\{b_{n}\right\}_{n \rightarrow \infty}$ is a major sequence of points in the sequence of outcomes $\left\{B_{n}\right\}_{n \rightarrow \infty}$ iff each $b_{n}$ is a point in $B_{n}$ and the sequence of points $\left\{b_{n}\right\}_{n \rightarrow \infty}$ has a magnitude that is equal to the greatest magnitude of any sequence that can be selected from the outcomes $B_{n}$. Formally, $b_{n} \in B_{n} \forall n$ and $\lim _{n \rightarrow \infty} \log \left(P\left[b_{n} \mid n \tau_{n}\right]\right) / n=$ $\lim _{n \rightarrow \infty} \max _{y \in B_{n}} \log \left(P\left[y \mid n \tau_{n}\right]\right) / n$. See Section 3 in Myerson [27] for a more detailed account of sequences of outcomes in LPG.
} 
This theorem states a simple technique to compute magnitudes of outcomes are defined by a finite series of inequalities.

A simple application is to compute the magnitude of the outcome $\{s(i) \geq s(j)\}$ for any pair of candidates $i$ and $j$.

Corollary 9. For any pair of candidates $i$ and $j,:$

$$
\mu[\{s(i) \geq s(j)\}]= \begin{cases}0 & \text { if } \rho(i)>\rho(j), \\ \mu[s(i)=s(j)] & \text { if } \rho(i) \leq \rho(j) .\end{cases}
$$

Proof. As previously defined, $\mathcal{C}_{k}$ stands for the set of ballots in which candidate $k$ is approved. For any pair $i, j$, we let $\rho_{i, j}$ the expected number of voters who approve of $i$ and not $j$ so that $\rho_{i, j}=\sum_{c \in \mathcal{C}_{i} \backslash \mathcal{C}_{j}} \tau(c)$. It follows that $\rho_{i}>\rho(j)$ is equivalent to $\rho_{i, j}>\rho_{j, i}$. Moreover, the outcome $\{s(i) \geq s(j)\}$ is equivalent to $B=\left\{\sum_{c \in \mathcal{C}_{i} \backslash \mathcal{C}_{j}} x(c)-\right.$ $\left.\sum_{c \in \mathcal{C}_{j} \mid \mathcal{C}_{i}} x(c) \geq 0\right\}$. Theorem 2 implies that the magnitude equals the optimal value of $\min _{X} \rho_{i, j} X+\rho_{j, i} X^{-1}-\rho_{i, j}-\rho_{j, i}$ provided that $X \geq 1$. Its minimum is reached when $X=\sqrt{\frac{\rho_{j, i}}{\rho_{i, j}}}$. Hence, whenever $\rho_{i, j}>\rho_{j, i} \Longleftrightarrow \rho(i)>\rho(j)$, the magnitude equals 0 . Otherwise, the magnitude equals $-\left(\sqrt{\rho_{i, j}}-\sqrt{\rho_{j, i}}\right)^{2}$, the magnitude of $\{s(i)=s(j)\}$, proving the claim.

The Magnitude Equivalence Theorem or MET (Núñez [33]) substantially reduces the computations of the magnitude of a pivot outcome: it allows us to use directly the DMT to compute magnitudes of pivot outcomes.

The DMT is conceived to compute the magnitude of outcomes defined by a series of inequalities involving the vote profile $x=(x(c))_{c \in C}$. Formally, using the $D M T$ we compute the magnitude of an outcome $B \subset Z(C)$ defined by

$$
B=\left\{\sum_{c \in C} a_{k}(c) x(c) \geq 0 \forall k \in J\right\} .
$$

However, a pivot outcome does not have this geometrical structure, i.e. for some $Y \subset K$, an outcome pivot $(Y)$ under $A V$ is defined by

$$
\begin{aligned}
& \forall y \in Y, s(y) \geq \max _{k \in K} s(k)-1 \\
& \forall k \notin Y, s(k) \leq \max _{k \in K} s(k)-2 .
\end{aligned}
$$

Given that the components $s(k)$ of the score profile $s$ are sums of the components $x(c)$ of the vote profile $x$, i.e. $s(k)=\sum_{c \in \mathcal{C}_{k}} x(c)$, we cannot express a pivot outcome 
only using linear inequalities involving $x$.

The MET shows that the magnitude of a pivot outcome coincides with the magnitude of an outcome than can be defined uniquely using this type of inequalities. The proof of the theorem is provided in the appendix.

Theorem 3 (Magnitude Equivalence Theorem, Núñez [33]). Let $Y$ be a subset of $K$ and pivot $(Y)$ be its associated pivot outcome. Given a large equilibrium sequence $\left(\sigma_{n}\right)_{n \rightarrow \infty}$,

$$
\mu[\operatorname{pivot}(Y)]=\mu[D],
$$

for some outcome $D \in Z(C)$ defined by

$$
D=\{s(k)=s(l) \forall k, l \in Y\} \cap\{s(k) \geq s(l) \forall k \in Y \text { and } l \in K \backslash Y\} .
$$

This result shows that there exists an outcome, defined by a series of inequalities depending on the vote profile $x$, which magnitude coincides with the magnitude of the pivot outcome. Indeed, the outcome $D$ defined by Theorem 3 can be written down as:

$$
D=\left\{\sum_{c \in C} a_{k}(c) x(c) \geq 0 \forall k \in J\right\},
$$

for some parameters $a_{k}$ as, by definition,

$$
s(k)=\sum_{c \in \mathcal{C}_{k}} x(c) .
$$

Thus, one can directly use the DMT to compute the magnitude of pivot outcomes, solving a simple constrained maximization problem. 